\title{
LOCAL UNIVERSALITY FOR ZEROS AND CRITICAL POINTS OF MONOCHROMATIC RANDOM WAVES
}

\author{
YAIZA CANZANI AND BORIS HANIN
}

\begin{abstract}
This paper concerns the asymptotic behavior of zeros and critical points for monochromatic random waves $\phi_{\lambda}$ of frequency $\lambda$ on a compact, smooth, Riemannian manifold $(M, g)$ as $\lambda \rightarrow \infty$. We prove global variance estimates for the measures of integration over the zeros and critical points of $\phi_{\lambda}$. These global estimates hold for a wide class of manifolds - for example when $(M, g)$ has no conjugate points and rely on new local variance estimates on zeros and critical points of $\phi_{\lambda}$ in balls of radius $\approx \lambda^{-1}$ around a fixed point. Our local results hold under conditions about the structure of geodesics that are generic in the space of all metrics on $M$.
\end{abstract}

\section{INTRODUCTION}

This article gives new local and global results about the measures of integration over the zero and critical point sets of a monochromatic random wave $\phi_{\lambda}$ of frequency $\lambda$. To introduce our results, let $(M, g)$ be a compact, smooth, Riemannian manifold without boundary of dimension $n \geq 2$, and write $\Delta_{g}$ for the positive definite Laplace-Beltrami operator. Consider an orthonormal basis $\left\{\varphi_{j}\right\}_{j=1}^{\infty}$ of $L^{2}(M, g)$ consisting of real-valued eigenfunctions $\Delta_{g} \varphi_{j}=\lambda_{j}^{2} \varphi_{j}$ with $0=\lambda_{0}<\lambda_{1} \leq \lambda_{2} \leq \cdots \nearrow \infty$, normalized so that $\left\|\varphi_{j}\right\|_{2}=1$. Monochromatic random waves of frequency $\lambda$ are Gaussian fields on $M$ defined by

$$
\phi_{\lambda}:=\frac{1}{\sqrt{\operatorname{dim}\left(H_{\lambda}\right)}} \sum_{\lambda_{j} \in[\lambda, \lambda+1]} a_{j} \varphi_{j}
$$

where the coefficients $a_{j} \sim N(0,1)$ are real valued i.i.d standard Gaussians and

$$
H_{\lambda}:=\bigoplus_{\lambda_{j} \in[\lambda, \lambda+1]} \operatorname{ker}\left(\Delta_{g}-\lambda_{j}^{2}\right)
$$

We write

$$
\phi_{\lambda} \in \mathrm{RW}_{\lambda}(M, g)
$$

for short. The ensembles $\phi_{\lambda}$ are Gaussian models for eigenfunctions of the Laplacian with eigenvalue approximately equal to $\lambda^{2}$ on a compact Riemannian manifold $(M, g)$. In the setting of a general smooth manifold the ensembles $\mathrm{RW}_{\lambda}$ were first defined by Zelditch in [32]. Zelditch was inspired in part by the influential work of Berry [5], which proposes that random waves on Euclidean space and flat tori are good semiclassical models for high frequency wavefunctions in quantum systems whose classical dynamics are chaotic.

Specifically, Berry proposed his Random Wave Conjecture: at a fixed $x \in M$ the local behavior of deterministic eigenfunctions $\varphi_{j}(x+u / \lambda)$ should be well-approximated, 
as $\lambda \rightarrow \infty$, by the behavior of frequency 1 random waves on $T_{x} M \cong \mathbb{R}^{n}$, so long as geodesics on $(M, g)$ are chaotic. The idea is that the rescaled eigenfunctions $\varphi_{j}(x+u / \lambda)$ approximately solve $\Delta f=f$ for the "frozen" constant coefficient Laplacian on $T_{x} M \cong \mathbb{R}^{n}$ and hence are almost frequency 1 eigenfunctions of $\Delta$ on $\mathbb{R}^{n}$ (see (17)). Moreover, since a classical free particle on $(M, g)$ moves along geodesics, in the long time (i.e. high energy) limit, it will become equidistributed on $M$ with respect to the volume form of $g$ if the geodesics on $(M, g)$ are chaotic. Hence, its quantum analogs, the eigenfunctions $\varphi_{j}$ as $\lambda_{j} \rightarrow \infty$, should also become delocalized and have no preferred points or directions on $M$, suggesting they are locally well approximated by Euclidean random waves.

Berry's Random Wave Conjecture has remained out of reach for deterministic sequences of eigenfunctions. However, the authors' previous work [7, 8] can be viewed as a weak form of the conjecture: the statement that, under some assumptions on the structure of geodesics on $(M, g)$, rescaled random waves $\phi_{\lambda}(x+u / \lambda)$ on $(M, g)$ have Berry's random waves on $\mathbb{R}^{n}$ as their local limits in the sense of $C^{\infty}$ convergence of covariance functions of Gaussian fields (see Definition 1 and Remark 3). This statement is non-trivial since it implies that the global geometry and topology of $(M, g)$ does not affect the local behavior of high frequency random waves.

The purpose of the present work is to derive several concrete results about the nodal sets and critical points of random waves from the kernel convergence in [7, 8. This point of view has been taken up in a number of articles (c.f. e.g. 22] and Section 1.1). Define the measures of integration over $\left\{\phi_{\lambda}=0\right\}$ and $\left\{d \phi_{\lambda}=0\right\}$ by

$$
Z_{\lambda}(\psi):=\int_{\phi_{\lambda}^{-1}(0)} \psi(x) d \mathcal{H}^{n-1}(x) \quad \text { and } \quad \operatorname{Crit}_{\lambda}(\psi):=\sum_{d \phi_{\lambda}(x)=0} \psi(x),
$$

where $\psi: M \rightarrow \mathbb{R}$ and $\mathcal{H}^{n-1}$ is the $(n-1)$-dimensional Hausdorff measure. Our main result gives asymptotics for the expected value and estimates for the variance of the linear statistics of $Z_{\lambda}$, Crit ${ }_{\lambda}$ that are valid for generic Riemannian metrics on $M$. For our estimates about the means of $Z_{\lambda}(\psi), \operatorname{Crit}_{\lambda}(\psi)$ we ask that $(M, g)$ be a manifold of isotropic scaling (see Definition 1 and Section 1.5). In particular, it is true for any manifold with negative curvature, or with no conjugate points. For our more delicate variance estimates to hold, we ask in addition that the restrictions of $\phi_{\lambda}$ to small balls centered at different points become asymptotically uncorrelated. Namely, we say that the random waves $\phi_{\lambda}$ have short-range correlations if for each $\varepsilon>0$ and every $\alpha, \beta \in \mathbb{N}$

$$
\sup _{\left\{x, y: d_{g}(x, y) \geq \lambda^{-1+\varepsilon}\right\}}\left|\nabla_{x}^{\alpha} \nabla_{y}^{\beta} \Pi_{\lambda}(x, y)\right|=o_{\varepsilon}\left(\lambda^{\alpha+\beta}\right),
$$

as $\lambda \rightarrow \infty$, where $\nabla_{x}, \nabla_{y}$ are covariant derivatives, and $\Pi_{\lambda}(x, y):=\operatorname{Cov}\left(\phi_{\lambda}(x), \phi_{\lambda}(y)\right)$ is the two point correlation function for $\phi_{\lambda}$, which is the kernel for the orthogonal projection onto $H_{\lambda}$. This condition is again generic in the space of Riemannian metrics on $(M, g)$ and is satisfied for example if for any pair of points $x, y \in M$ the measure of geodesic arcs joining them is zero. This is known to happen on manifolds of negative curvature, or more generally, with no conjugate points. We refer the reader to Section 1.5 for a discussion of when $(M, g)$ is a manifold with isotropic scaling satisfying (2). 
We are ready to sate our main theorem. It is the first result that gives variance estimates for $Z_{\lambda}$, Crit ${ }_{\lambda}$ that holds on a large class of smooth Riemannian manifolds (we refer the reader to Section 1.1 for a discussion of previous work). In what follows $d v_{g}$ is the Riemannian volume form.

Theorem 1. Let $(M, g)$ be a smooth, compact, Riemannian manifold of dimension $n \geq 2$ with no boundary. Let $\phi_{\lambda} \in \mathrm{RW}_{\lambda}(M, g)$ and suppose that $M$ is a manifold of isotropic scaling (Definition 1). Then, for any bounded measurable function $\psi: M \rightarrow$ $\mathbb{R}$,

$$
\lim _{\lambda \rightarrow \infty} \mathbb{E}\left[\lambda^{-1} Z_{\lambda}(\psi)\right]=\frac{1}{\sqrt{\pi n}} \frac{\Gamma\left(\frac{n+1}{2}\right)}{\Gamma\left(\frac{n}{2}\right)} \int_{M} \psi(x) d v_{g}(x),
$$

and

$$
\lim _{\lambda \rightarrow \infty} \mathbb{E}\left[\lambda^{-n} \operatorname{Crit}_{\lambda}(\psi)\right]=C_{n} \int_{M} \psi(x) d v_{g}(x),
$$

where $C_{n}$ is a positive constant that depends only on $n$. Suppose further that $\phi_{\lambda}$ has short-range correlations in the sense of (2). Then,

$$
\operatorname{Var}\left[\lambda^{-1} Z_{\lambda}(\psi)\right]=O\left(\lambda^{-\frac{n-1}{2}}\right)
$$

and

$$
\operatorname{Var}\left[\lambda^{-n} \operatorname{Crit}_{\lambda}(\psi)\right]=O\left(\lambda^{-\frac{n-1}{2}}\right)
$$

as $\lambda \rightarrow \infty$.

Remark 1. The test function $\psi$ can be replaced by a function

$$
\psi(x)=\psi\left(x, \phi_{\lambda}(x), D^{2} \phi_{\lambda}(x), \ldots\right)
$$

depending on the jets of $\phi_{\lambda}$ provided $\psi: \mathbb{R}^{n} \times C^{0}\left(\mathbb{R}^{n}, \mathbb{R}^{k}\right) \rightarrow \mathbb{R}$ is bounded and continuous when $C^{0}\left(\mathbb{R}^{n}, \mathbb{R}^{k}\right)$ is equipped with the topology of uniform convergence on compact sets. Hence, for example, we could study the distribution of critical values by taking

$$
\psi\left(u, \phi_{\lambda}\right)=\mathbf{1}_{\left\{\phi_{\lambda}^{x} \geq \alpha\right\}}(u), \quad \alpha \in \mathbb{R} .
$$

Remark 2. The proof of Theorem 1 actually shows that (3) holds as soon as almost every point is a point of isotropic scaling. That is, it holds provided $\operatorname{vol}_{g}(M \backslash \mathcal{I} \mathcal{S}(M, g))=$ 0 (see Definition 11). Also, by the Borel-Cantelli Lemma, if $n \geq 4$ and $\phi_{j}$ are independent frequency $j \in \mathbb{N}$ random waves on $(M, g)$, then (5) shows that the total nodal set measure $j^{-1} Z_{j}(\psi)-\mathbb{E}\left[j^{-1} Z_{j}(\psi)\right]$ converges almost surely to 0 . Finally, when $n=2$ we have $C_{2}=\mathbb{E}\left[\right.$ Crit $\left._{\infty, 1}\right]=\frac{1}{4 \pi \sqrt{6}}$ where $C_{2}$ is the dimensional constant in (4).

Theorem 1 1hinges on a careful study of the statistics of $\phi_{\lambda}$ when restricted to "wavelength balls" of radius $\approx \lambda^{-1}$ around a fixed point $x \in M$ of isotropic scaling. The results that describe the behavior of $Z_{\lambda}$ or Crit ${ }_{\lambda}$ restricted to these shrinking balls are given in Sections 1.3 and 1.4 respectively. Before these two sections we give an overview of the prior results in Section 1.1 and give a formal definition of points of isotropic scaling in Section 1.2 . 
1.1. Prior results. To the best of our knowledge, Theorem 1 is the first result with a non-trivial variance estimate for the Hausdorff measure of the nodal set of random waves for a generic smooth Riemannian manifold (for real analytic $(M, g)$ a weaker estimate was given in [32, Cor. 2]). A version of (3) was also stated, with a heuristic proof, in [32, Prop. 2.3] for both Zoll and aperiodic manifolds.

Previous results on the Hausdorff measure of nodal sets focus primarily on exactly solvable examples, where more precise variance estimates are available. In these settings, due to the degeneracy of the spectrum of the Laplacian, one replaces random waves by random exact eigenfunctions. On round spheres, for instance, Bérard [4] proved (3) (example (1) on p.3). Later, in the same setting, Neuheisel 24] and Wigman [30] obtained upper bounds for the variance that are of polynomial order in $\lambda$. Further, on $S^{2}$, Wigman 31 found that the variance actually grows like $\lambda^{-2} \log \lambda$ as $\lambda \rightarrow \infty$, much better than the general $O\left(\lambda^{-1 / 2}\right)$ estimate in (5). On flat tori $\mathbb{T}^{n}$ (for exact eigenfunctions) Rudnick and Wigman [26] computed the expected value of the total Hausdorff measure of the zero set and gave an upper bound of the form $\lambda^{2}\left(\operatorname{dim}\left(H_{\lambda}\right)\right)^{-1 / 2}$ on its variance. Subsequently, on $\mathbb{T}^{2}$, Krishnapur, Kurlberg and Wigman [19] found that the variance is asymptotic to a constant, while Marinucci, Pecatti, Rossi and Wigman proved that the size of the zero set converges to a limiting distribution that is not Gaussian and depends on the angular distribution of lattice points on circles [21.

The behavior of the number of critical points has been studied in detail on $S^{2}$. Nicolaescu [25] studied the expected value of the number of critical points, obtaining (4). The variance was studied by Cammarota, Marinucci and Wigman [10]. They obtain a polynomial upper bound. This upper bound was later improved by Cammarota and Wigman [9] who proved that the variance grows like $\lambda^{2} \log \lambda$ (as opposed to our $\lambda^{7 / 2}$ estimate) as $\lambda \rightarrow \infty$. Finally, for a smooth domain in $\mathbb{R}^{2}$, Nourdin-Peccati-Rossi [22] prove that both for real and complex random waves, the Hausdorff measure of the nodal set is asymptotically normal in the high frequency limit.

1.2. Isotropic scaling. Our main result, Theorem 1, hinges on understanding the statistics, as $\lambda \rightarrow \infty$, of $\phi_{\lambda}$ restricted to "wavelength balls" of radius $\approx \lambda^{-1}$ around a fixed point $x \in M$. After rescaling by $1 / \lambda$, the function $\phi_{\lambda}$ has frequency approximately 1 on such balls in the sense that it also the approximate local eigenvalue equation

$$
\Delta_{T_{x} M} \phi_{\lambda}\left(x+\frac{u}{\lambda}\right) \approx \phi_{\lambda}\left(x+\frac{u}{\lambda}\right),
$$

where $\Delta_{T_{x} M}$ denotes the flat Laplacian on the tangent space at $T_{x} M$. Moreover, as $\lambda \rightarrow \infty$, for a generic Riemannian metric on $M$, the covariance kernels $\Pi_{\lambda}$ of $\phi_{\lambda}$ converge in the $C^{\infty}$ topology to those of a limiting ensemble of frequency 1 functions $\phi_{\infty}$, called frequency 1 random waves on $\mathbb{R}^{n} \cong T_{x} M$, where $n$ is the dimension of $M$. We explain this in what follows.

It is natural to study $\phi_{\lambda}$ by fixing $x \in M$ and considering the rescaled pullback of $\phi_{\lambda}$ to the tangent space $T_{x} M$. We denote this pullback by

$$
\phi_{\lambda}^{x}(u):=\phi_{\lambda}\left(\exp _{x}\left(\frac{u}{\lambda}\right)\right) .
$$


The law of $\phi_{\lambda} \in \mathrm{RW}_{\lambda}(M, g)$, which is a centered smooth Gaussian field, is determined by its covariance kernel

$$
\Pi_{\lambda}(x, y):=\operatorname{Cov}\left(\phi_{\lambda}(x), \phi_{\lambda}(y)\right)=\frac{1}{\operatorname{dim} H_{\lambda}} \sum_{\lambda_{j} \in[\lambda, \lambda+1]} \varphi_{j}(x) \varphi_{j}(y),
$$

$x, y \in M$. The function $\Pi_{\lambda}(x, y)$ is the Schwartz kernel for the spectral (orthogonal) projector $\Pi_{[\lambda, \lambda+1]}: L^{2}(M, g) \rightarrow H_{\lambda}$, normalized to have unit trace. The dilated functions $\phi_{\lambda}^{x}$ are centered Gaussian fields on $T_{x} M$, and we denote their scaled covariance kernel by

$$
\Pi_{\lambda}^{x}(u, v):=\operatorname{Cov}\left(\phi_{\lambda}^{x}(u), \phi_{\lambda}^{x}(v)\right)=\Pi_{\lambda}\left(\exp _{x}\left(\frac{u}{\lambda}\right), \exp _{x}\left(\frac{v}{\lambda}\right)\right),
$$

$u, v \in T_{x} M$. When $x$ is a point of isotropic scaling (see Definition 1 below), the kernels $\Pi_{\lambda}^{x}$ converge in the $C^{\infty}$ sense to the covariance kernel of a limiting ensemble of random functions

$$
\phi_{\infty}^{x} \in \mathrm{RW}_{1}\left(T_{x} M, g_{x}\right),
$$

called frequency 1 random waves on $\mathbb{R}^{n} \cong T_{x} M$. Here $g_{x}$ denotes the constant coefficient metric obtained by "freezing" $g$ at $x$. The random wave $\phi_{\infty}^{x}$ is defined as the unique centered Gaussian field with covariance kernel

$$
\Pi_{\infty}^{x}(u, v)=(2 \pi)^{\frac{n}{2}} \frac{J_{\frac{n-2}{2}\left(|u-v|_{g_{x}}\right)}}{|u-v|_{g_{x}}^{\frac{n-2}{2}}}=\int_{S_{x} M} e^{i\langle u-v, \omega\rangle_{g_{x}}} d \omega .
$$

Here $J_{\nu}$ denotes a Bessel function of the first kind with index $\nu, S_{x} M$ is the unit sphere in $T_{x} M$ with respect to $g_{x}$, and $d \omega$ is the hypersurface measure. The formal definition is the following.

Definition 1. A point $x \in M$ is a point of isotropic scaling, denoted $x \in \mathcal{I S}(M, g)$, if for every non-negative function $r_{\lambda}$ satisfying $r_{\lambda}=o(\lambda)$ as $\lambda \rightarrow \infty$ and all $\alpha, \beta \in \mathbb{N}^{n}$, we have

$$
\sup _{u, v \in B_{r_{\lambda}}}\left|\partial_{u}^{\alpha} \partial_{v}^{\beta}\left[\Pi_{\lambda}^{x}(u, v)-\Pi_{\infty}^{x}(u, v)\right]\right|=o_{\alpha, \beta}(1)
$$

as $\lambda \rightarrow \infty$, where the rate of convergence depends on $\alpha, \beta$ and $B_{R}$ denotes a ball of radius $R$ centered at $0 \in T_{x} M$. We also say that $M$ is a manifold of isotropic scaling if $M=\mathcal{I S}(M, g)$ and if the convergence in (11) is uniform over $x \in M$ for each $\alpha, \beta \in \mathbb{N}^{n}$.

Remark 3. If the set of geodesic loop directions $\mathcal{L}_{x, x} \subset S_{x}^{*} M$ through $x$ has measure 0 , then $x \in \mathcal{I S}(M, g)$ by [8, Thm. 1]. This implication also holds if the spectral interval $[\lambda, \lambda+1]$ in the definition of $\phi_{\lambda}$ is replaced by $[\lambda, \lambda+\eta(\lambda)]$ with $\eta(\lambda)=o(\lambda)$ and $\liminf \operatorname{in}_{\lambda \rightarrow \infty} \eta(\lambda)>0$. Even for these more general spectral windows, the condition that $M$ is a manifold of isotropic scaling is generic in the space of Riemannian metrics on any smooth compact manifold. See (21) and Section 1.5] for details. By [29, Lem 6.1], the condition that $\left|\mathcal{L}_{x, x}\right|=0$ for all $x \in M$ is generic in the space of Riemannian metrics on a fixed compact smooth manifold $M$, i.e. holds away from a countable union of nowhere dense sets. 
In addition, it is very likely that if $(M, g)$ has no conjugate points, then the condition

$$
\lim _{\lambda \rightarrow \infty} \log (\lambda) \cdot \eta_{\lambda}=\infty
$$

implies $\mathcal{I} \mathcal{S}(M, g)=M$. This was proved by B. Keeler in [18, but with the convergence in (11) only holding for $\alpha=\beta=0$. This involves a non-trivial off-diagonal extension of Bérard's estimates in [2, 3].

If $x \in \mathcal{I} \mathcal{S}(M, g)$, then in any coordinates around $x$ for which $g_{x}=\mathrm{Id}$, the scaling limit of waves in $\operatorname{RW}_{\lambda}(M, g)$ around $x$ is universal in the sense that it depends only on the dimension of $M$. In the language of Nazarov-Sodin [23] the asymptotics (11) imply that if $M=\mathcal{I} \mathcal{S}(M, g)$, then the ensembles $\operatorname{RW}_{\lambda}(M, g)$ have translation invariant local limits. For ensembles with such translation invariant local limits, Nazarov-Sodin [23], Sarnak-Wigman [28, Gayet-Welschinger [13, 14, 15] and Canzani-Sarnak [11, as well as others, prove very interesting results on non-integral statistics of the nodal sets of random waves. Such nodal set statistics include the number of connected components, Betti numbers, and topological types. A key step in all these articles, however, is to find some way to reduce the study of non-integral statistics to integral statistics, such as the volume of the zero set or the number of critical points. Thus, the results on integral statistics in this article may be useful for future work on non-integral nodal set statistics of random waves as well.

1.3. Local universality of zeros. Our first result concerns the behavior of the nodal set of the rescaled random wave $\phi_{\lambda}^{x}$ for $x \in \mathcal{I} S(M, g)$ (see Definition 1). Let us denote by $Z_{\lambda}^{x}$ its Riemannian hypersurface (i.e. Hausdorff) measure:

$$
Z_{\lambda}^{x}(A):=\mathcal{H}^{n-1}\left(\left(\phi_{\lambda}^{x}\right)^{-1}(0) \cap A\right), \quad \forall A \subseteq T_{x} M \text { measurable. }
$$

Theorem 2 concerns the restriction of $Z_{\lambda}^{x}$ to various balls $B_{r}$ of radius $r$ centered at $0 \in T_{x} M$. We set

$$
Z_{\lambda, r}^{x}:=\frac{\mathbf{1}_{B_{r}} \cdot Z_{\lambda}^{x}}{\operatorname{vol}\left(B_{r}\right)} \quad \text { and } \quad Z_{\infty, r}^{x}:=\frac{\mathbf{1}_{B_{r}} \cdot Z_{\infty}^{x}}{\operatorname{vol}\left(B_{r}\right)} .
$$

We have denoted by $\mathbf{1}_{B_{r}}$ the characteristic function of the ball $B_{r}$ and by $Z_{\infty}^{x}$ the hypersurface measure on $\left(\phi_{\infty}^{x}\right)^{-1}(0)$ for $\phi_{\infty}^{x} \in \mathrm{RW}_{1}\left(T_{x} M, g_{x}\right)$. For various measures $\mu$, we write $\mu(\psi)$ for integration of a measurable function $\psi$ against $\mu$. In particular,

$$
Z_{\lambda, r}^{x}(1)=\frac{\mathcal{H}^{n-1}\left(\left(\phi_{\lambda}^{x}\right)^{-1}(0) \cap B_{r}\right)}{\operatorname{vol}\left(B_{r}\right)} .
$$

Theorem 2 (Weak Convergence of Zero Set Measures). Let $(M, g)$ be a smooth, compact, Riemannian manifold of dimension $n \geq 2$ with no boundary. Fix a non-negative function $r_{\lambda}$ that satisfies $r_{\lambda}=o(\lambda)$ as $\lambda \rightarrow \infty$. Let $\phi_{\lambda} \in \mathrm{RW}_{\lambda}(M, g)$ and $x \in \mathcal{I} \mathcal{S}(M, g)$. Suppose $\lim _{\lambda \rightarrow \infty} r_{\lambda}$ exists and equals $r_{\infty} \in(0, \infty]$.

Case $1\left(r_{\infty}<\infty\right)$ : The measures $Z_{\lambda, r_{\lambda}}^{x}$ converge to $Z_{\infty, r_{\infty}}^{x}$ weakly in distribution. That is, for any bounded, measurable function $\psi: T_{x} M \rightarrow \mathbb{R}$

$$
Z_{\lambda, r_{\lambda}}^{x}(\psi) \stackrel{d}{\longrightarrow} Z_{\infty, r_{\infty}}^{x}(\psi)
$$


as $\lambda \rightarrow \infty$, where $\stackrel{d}{\longrightarrow}$ denotes convergence in distribution.

Case $2\left(r_{\infty}=\infty\right)$ : We have the following convergence in probability to a constant:

$$
Z_{\lambda, r_{\lambda}}^{x}(1) \stackrel{p}{\longrightarrow} \quad \frac{1}{\sqrt{\pi n}} \frac{\Gamma\left(\frac{n+1}{2}\right)}{\Gamma\left(\frac{n}{2}\right)},
$$

as $\lambda \rightarrow \infty$. In particular,

$$
\lim _{\lambda \rightarrow \infty} \operatorname{Var}\left[Z_{\lambda, r_{\lambda}}^{x}(1)\right]=0 .
$$

After posting an early version of this article, G. Peccati brought to our attention that the convergence in distribution (13) for the nodal set measure $Z_{\lambda, r_{\lambda}}^{x}$ in balls of size $R / \lambda$ can be obtained directly from the $C^{\infty}$ scaling asymptotics of the covariance function. He kindly allowed us to include his argument, which we reproduce in the proof of Theorem 2 when $r_{\infty}<\infty$ (see Section 3.1).

Remark 4. Just as in Remark 1, the function $\psi$ in (13) can be allowed to depend on the jets $D^{j} \phi_{\lambda}, j \geq 1$. More precisely, $\psi(u)$ can be replaced by $\psi(u, W(u))$, where $W$ is a random field so that $u \mapsto\left(\phi_{\lambda}^{x}(u), W(u)\right)$ is a continuous Gaussian field with values in $\mathbb{R}^{1+k}$ and $\psi: \mathbb{R}^{n} \times C^{0}\left(\mathbb{R}^{n}, \mathbb{R}^{k}\right) \rightarrow \mathbb{R}$ is bounded and continuous when $C^{0}\left(\mathbb{R}^{n}, \mathbb{R}^{k}\right)$ is equipped with the topology of uniform convergence on compact sets. Since $\left(\phi_{\lambda}^{x}(u), D \phi_{\lambda}^{x}(u), D^{2} \phi_{\lambda}^{x}(u), \ldots\right)$ is a smooth Gaussian field, we may take $W(u)=\left(D^{j} \phi_{\lambda}(u), j \geq 1\right)$. Similarly, in (14) and (15)), the function $1=1(u)$ can be replaced by $\psi(W(u))$ where again $\psi: C^{0}\left(\mathbb{R}^{n}, \mathbb{R}^{k}\right) \rightarrow \mathbb{R}$ is bounded and continuous in the topology of uniform convergence on compact sets. The only difference is that (14) then reads

$$
Z_{\lambda, r_{\lambda}}^{x}(\psi)-\mathbb{E}\left[Z_{\infty, r_{\lambda}}^{x}(\psi)\right] \stackrel{p}{\longrightarrow} 0 .
$$

Remark 5. The relations (14) and (15) hold even if the balls $B_{r_{\lambda}}$ in the definition of $Z_{\lambda, r_{\lambda}}^{x}$ are replaced by any $\lambda$-dependent sets $A_{\lambda, r_{\lambda}}$ for which the diameter is bounded above and below by a constant times $r_{\lambda}$, and whose volume tends to infinity when $r_{\lambda} \rightarrow \infty$.

Remark 6. The rates of convergence in (13)-(15) - even after the generalizations indicated in Remarks 4 and 5 - are uniform as $x$ varies over a compact set $S \subset \mathcal{I S}(M, g)$ as long as the convergence in (11) is uniform over $S$.

Theorem 2 is proved in Section 2.1 and Section 3 .

1.4. Local universality of critical points. We state in this section our results on critical points of random waves, which have been extensively studied (c.f. e.g., [9, 10, 25]). Let $x \in M$ and for each $r>0$ define the normalized counting measure

$$
\operatorname{Crit}_{\lambda, r}^{x}:=\frac{1}{\operatorname{vol}\left(B_{r}\right)} \sum_{\substack{d \phi_{\lambda}^{x}(u)=0 \\ u \in B_{r}}} \delta_{u}
$$

of critical points in a ball of radius $r$. We define Crit $_{\infty, r}^{x}$ in the same way as Crit ${ }_{\lambda, r}^{x}$ but with $\phi_{\lambda}^{x}$ replaced by $\phi_{\infty}^{x} \in \mathrm{RW}_{1}\left(T_{x} M, g_{x}\right)$, and continue to write $\mu(\psi)$ for the pairing 
of a measure $\mu$ with a function $\psi$. For example,

$$
\operatorname{Crit}_{\lambda, r}^{x}(1)=\frac{\#\left\{u \in B_{r}: d \phi_{\lambda}^{x}(u)=0\right\}}{\operatorname{vol}\left(B_{r}\right)} .
$$

Theorem 3. Let $(M, g)$ be a smooth, compact, Riemannian manifold of dimension $n \geq 2$ with no boundary. Fix a non-negative function $r_{\lambda}$ that satisfies $r_{\lambda}=o(\lambda)$ as $\lambda \rightarrow \infty$. Let $\phi_{\lambda} \in \mathrm{RW}_{\lambda}(M, g)$ and $x \in \mathcal{I S}(M, g)$. Suppose that $\lim _{\lambda \rightarrow \infty} r_{\lambda}$ exists and equals $r_{\infty} \in(0, \infty]$.

Case 1. $\left(r_{\infty}<\infty\right)$ : For $k=1,2$ and each bounded measurable function $\psi: T_{x} M \rightarrow \mathbb{R}$

$$
\lim _{\lambda \rightarrow \infty} \mathbb{E}\left[\operatorname{Crit}_{\lambda, r_{\lambda}}^{x}(\psi)^{k}\right]=\mathbb{E}\left[\operatorname{Crit}_{\infty, r_{\infty}}^{x}(\psi)^{k}\right] .
$$

Case 2. $\left(r_{\infty}=\infty\right)$ : We have

$$
\lim _{\lambda \rightarrow \infty} \operatorname{Var}\left[\operatorname{Crit}_{\lambda, r_{\lambda}}^{x}(1)\right]=\mathbb{E}\left[\operatorname{Crit}_{\infty, 1}^{x}(1)\right] .
$$

This limit is the expected number of critical points in a ball of radius 1 for frequency 1 random waves on $\mathbb{R}^{n}$, which is independent of $x$.

Remark 7. We prove in Section 2.3 that the moments $\mathbb{E}\left[\left(\operatorname{Crit}_{\infty, r_{\infty}}^{x}(\psi)\right)^{k}\right]$ are finite for $k=1,2$. In particular, we show in Section 3 that if $\operatorname{dim}(M)=2$, then $x \in M$

$$
\mathbb{E}\left[\operatorname{Crit}_{\infty, 1}^{x}(1)\right]=\frac{1}{4 \pi \sqrt{6}} \text {. }
$$

Also, just as in Remark 5 , the balls $B_{r_{\lambda}}$ in (18) can be replaced by any $\lambda$-dependent sets $A_{\lambda, r_{\lambda}}$ for which the diameter is bounded above and below by a constant times $r_{\lambda}$ and whose volume tends to infinity with $r_{\lambda}$.

Remark 8. Just as in Remark 1, both $\psi$ in (17) and the function 1 being integrated against Crit $_{\lambda, r_{\lambda}}^{x}$ in (18) can be replaced by a bounded continuous function of the jets of $\phi_{\lambda}$, giving information for instance about critical points filtered by critical value.

Remark 9. Just as in Remark 6, the rates of convergence in (17) and (18) - even after the generalizations indicated in Remaks 7 and 8 - are uniform over $x \in S \subset \mathcal{I S}(M, g)$ if (11) is uniform over $S$.

On the $n$-dimensional flat torus, Nicolaescu [25] obtained several results related to Theorem 3 in the $r_{\infty}<\infty$ case. We prove Theorem 3 in Section 2.2 and Section 3 ,

1.5. Sufficient conditions for isotropic scaling and short-range correlations. To apply Theorems 2 and 3 one must verify that some $x \in M$ belongs to $\mathcal{I} S(M, g)$, the points of isotropic scaling (Definition 11). This is typically difficult to do directly, but can be done by hand for eigenvalue windows of the form $[\lambda, \lambda+1]$ on simple examples, such as flat tori, by verifying a condition about the geodesics through $x$. Namely, for $x, y \in M$ denote by

$$
\mathcal{L}_{x, y}=\left\{\xi \in S_{x} M: \exists t>0 \text { s.t. } \exp _{x}(t \xi)=y\right\}
$$

the set of directions that generate geodesic arcs from $x$ to $y$. Here, $S_{x} M=\left\{\xi \in T_{x} M\right.$ : $\left.|\xi|_{g(x)}=1\right\}$ is the unit sphere in $T_{x} M$. Theorem 1 in [8] shows that

$$
\left|\mathcal{L}_{x, x}\right|=0 \quad \Longrightarrow \quad x \in \mathcal{I} S(M, g),
$$


where $\left|\mathcal{L}_{x, x}\right|$ denotes the volume of $\mathcal{L}_{x, x}$ inside $S_{x} M$. There is a similar sufficient condition for the short-range correlations assumption in Theorem 1 .

$$
\left|\mathcal{L}_{x, y}\right|=0 \quad \forall x, y \in M \quad \Longrightarrow \quad R W_{\lambda}(M, g) \text { have short-range correlations. }
$$

Indeed, when $\left|\mathcal{L}_{x, y}\right|=0$ for all $x, y \in M$ and any $\varepsilon>0$ [27, Thm. 3.3] gives that for all $P, Q$ pseudodifferential operators on $M$ of with $\operatorname{ord} P=\operatorname{ord} Q$,

$$
\sup _{x, y: d(x, y) \geq \varepsilon}\left|P_{x} Q_{y} \Pi_{\lambda}(x, y)\right|=o\left(\lambda^{\operatorname{ord} P+\operatorname{ord} Q}\right),
$$

as $\lambda \rightarrow \infty$. Here, the subscripts $x$ and $y$ indicate that $P$ and $Q$ are acting on the $x$ and $y$ variables, respectively. Note that $\Delta^{-\frac{m}{2}} \Pi_{\lambda}(x, y)=\lambda^{-\frac{m}{2}} R \Pi_{\lambda}(x, y)$ with $R$ being an order zero pseudodifferential operator for any $m \in \mathbb{N}$. If we have that $\operatorname{ord} P=\operatorname{ord} Q+m$ with $m \in \mathbb{N}$, then

$$
P Q \Pi_{\lambda}(x, y)=\lambda^{-\frac{m}{2}} P Q \Delta^{\frac{m}{2}} R \Pi_{\lambda}(x, y),
$$

and the result follows since $Q \Delta^{\frac{m}{2}} R$ has the same order as $P$. Combining (23) with Remark 3 after Theorem 2 in [8] yields (22).

By [29, Lem 6.1], the condition that $\left|\mathcal{L}_{x, x}\right|=0$ for all $x \in M$ is generic in the space of Riemannian metrics on a fixed compact smooth manifold $M$. It is likely that a similar argument would show that $\left|\mathcal{L}_{x, y}\right|=0$ for all $x, y \in M$ is also generic. It is known, however, that the condition that $\left|\mathcal{L}_{x, y}\right|=0$ holds for all $x, y \in M$ if $(M, g)$ is negatively curved or, more generally, has no conjugate points.

1.6. Novel aspects of the paper. As mentioned above, this article gives what appear to be the first variance estimates for $Z_{\lambda}$, Crit ${ }_{\lambda}$ that hold on a large class of smooth Riemannian manifolds. This is in contrast to the many articles mentioned in Section 1.1 that treat integrable models such as spheres and torii. The main new ingredients are the following. First, we use the spectral theory results in [7, 8, which show that the scaling limits of random waves at non-self focal points on $(M, g)$ are frequency 1 random waves on $\mathbb{R}^{n}$ (see (11)). We do not use the spectral theory results in our proofs directly, but the fact that for a generic metric on $M$ all points are non self-focal means that the techniques in this article apply to generic $(M, g)$. Second, we give several new arguments about frequency 1 random waves, which allow us to apply the Kac-Rice formula to zeros and critical points of frequency 1 random waves (see around (24) and Section 2 below). Finally, we give an essentially combinatorial argument for patching together our local variance estimates from Theorems 2 and 3 to obtain the global results in Theorem 1 (see Section 4).

Indeed, our method for studying zeros and critical points of random waves relies on the Kac-Rice formula. Many previous articles (e.g. [9, 10, 19, 24, 25, 26, 30, 31, 32]) use the Kac-Rice formula to study the expected value and variance of the size of zero sets and number of critical points for random waves on flat tori and round spheres. In the vast majority of these cases, the Kac-Rice formula is not used directly. Instead, the authors explain that they cannot verify the non-degeneracy or the 1-jet spanning hypotheses of the Kac-Rice Theorem (Theorem 21). They then use modified, or approximate, Kac-Rice formulae adapted to each setting. In some instances this is unavoidable because the non-degeneracy hypothesis (2) can fail globally in the presence of many symmetries. 
We prove in contrast that the Kac-Rice formula can be applied to study all moments of the zero and critical points sets of for frequency 1 random waves $\phi_{\infty}$ on $\mathbb{R}^{n}$. As we explained above, $\phi_{\infty}$ are the scaling limits of $\phi_{\lambda}$ in balls of shrinking radii around a fixed $x \in M$ as long as $x$ is a point of isotropic scaling (see Definition 1 and Section 2.1 - Section 2.2). To prove Theorems 2 and 3, we use the Kac-Rice formula to write integral expression for moments of the measures of integration over the zero and critical points sets of the limiting random waves $\phi_{\infty}$ in wavelength balls around $x$. For the first and second moments we are then able to check that we can apply the Kac-Rice formula directly for $\phi_{\lambda}^{x}$ when $\lambda$ is large but finite and that the $\lambda \rightarrow \infty$ limit of the resulting expressions are the original expressions for the limiting frequency one random waves on $T_{x} M$.

Proving that we can apply the Kac-Rice formula to study zeros and critical points of $\phi_{\infty}$ requires several new arguments that rely on analysis of frequency 1 functions (i.e. smooth functions in $\operatorname{ker}\left(\Delta_{\mathbb{R}^{n}}-1\right)$ ) on $\mathbb{R}^{n}$. For instance, combining Propositions 4 and 5, we find that frequency 1 functions separate 1 -jets. More precisely, given $m$ distinct points $u_{1}, \ldots, u_{m} \in \mathbb{R}^{n}$ and constants $\left\{\alpha_{i}, \beta_{i, j}, 1 \leq i \leq m, 1 \leq j \leq n\right\}$ there exists a smooth real-valued function $f \in \operatorname{ker}\left(\Delta_{\mathbb{R}^{n}}-1\right)$ such that

$$
f\left(u_{i}\right)=\alpha_{i} \quad \text { and } \quad \partial_{j} f\left(u_{i}\right)=\beta_{i, j} .
$$

If $f$ were allowed to be any smooth function, then such a result is straightforward. However, with the restriction that $f$ have frequency precisely 1 , we could not find such results in the literature. Let us also mention that after the writing of this article, it was explained to us by M. Sodin that there is a alternative complex analytic approach to proving such facts about frequency 1 functions.

This article also includes a new argument (see Section 4) for how to patch together local variance estimates for nodal and critical sets on balls of shrinking radii $\lambda^{-\varepsilon}$ to obtain quantitative upper bounds on the variance of the volumes of the zero and critical point sets of $\phi_{\lambda}$ (see (5) and (6) ). In "integrating" the local variance estimates, we control neither the rate at which the covariance kernels $\Pi_{\lambda}$ of the random waves $\phi_{\lambda}$ converge pointwise to their scaling limits near various $x \in M$ (see Definition 10 nor the rate at which off-diagonal correlations decay (see Definition 21). Nonetheless, we are able to obtain quantitative variance estimates by using lower bounds on the volume of the set of points $(x, y) \in M \times M$ at which the spectral projection (covariance) kernel $\Pi_{\lambda}(x, y)$ is already measurably small (of order $\lambda^{-\frac{n-1}{2}}$, where $n$ is the dimension of $M)$. This is the content of Section 4 and is related in spirit to the work of JakobsonPolterovich [17].

1.7. Acknowledgements. We are grateful to an anonymous referee for finding a gap in a previous version of this article and for significantly improving some aspects of the exposition. That version concerned a wide class of integral statistics (not just zeros and critical points) of monochromatic random waves. However, there was an error in the previous incarnation of what are now Propositions 4 and 5. The new propositions fix the mistake for the special cases of zeros and critical points. We leave the extension of the results in this paper to more general integral statistics for future work. The second author would also like to thank Damien Gayet and Thomas Letendre for several useful discussions pertaining to the arguments in Proposition 6 . 
1.8. Outline. The rest of our paper is organized as follows. First, in Section 2, we recall a variant of the Kac-Rice formula and prove that it can be applied to study all moments for the measures of integration over the zeros and critical points of frequency 1 random waves on $\mathbb{R}^{n}$. We then complete the proof of the our local results (Theorems 2 and 3) in Section 3. Finally, in Section 4, we explain how to use the assumption that random waves have short-range correlations on $(M, g)$ (see Definition 2) to prove our global results (Theorem 1).

\section{Analysis of Frequency 1 Random Waves on $\mathbb{R}^{n}$}

Let $\phi_{\infty}$ be a frequency 1 random wave on $\mathbb{R}^{n}$ (see (10)). We prove in this section that the following variant of the Kac-Rice formula (an amalgam of Azaïs-Wshebor [1. Thms. 6.2, 6.3, Props. 6.5,6.12]) can be applied to study all the moments for the measures of integration over its zero and critical point sets. In what follows we write $\mathcal{H}^{k}$ for the dimension $k$ Hausdorff measure on $\mathbb{R}^{n}$.

Kac-Rice Theorem. Let $U$ be an open subset of $\mathbb{R}^{n}$ and $X: U \rightarrow \mathbb{R}^{k}$ be a Gaussian field with $k \leq n$. Fix $m \in \mathbb{N}$, and suppose that

(1) $X$ is almost surely $C^{2}$.

(2) Non-degeneracy: For every collection of distinct points $\left\{u_{j}\right\}_{j=1}^{m}$ the Gaussian vector $\left(X\left(u_{j}\right)\right)_{j=1}^{m}$ has a non-degenerate distribution.

If $k<n$ suppose in addition that

(3) 1-Jet Spanning Property: For $u \in U$, the joint distribution of $X(u)$ and the Jacobian $\left(\partial_{i} X_{j}(u)\right)_{1 \leq i \leq n}$ is a non-degenerate Gaussian.

Then, if $k<n$,

$\mathbb{E}\left[\mathcal{H}^{n-k}(\{X=0\} \cap B)^{m}\right]=\int_{B^{m}} Y_{m, X}\left(u_{1}, \ldots, u_{m}\right) \operatorname{Den}_{X\left(u_{1}\right), \ldots, X\left(u_{m}\right)}(0, \ldots, 0) d u_{1} \ldots d u_{m}$

for every measurable Borel set $B \subseteq U$, where

$$
Y_{m, X}\left(u_{1}, \ldots, u_{m}\right)=\mathbb{E}\left[\prod_{j=1}^{m}\left[\operatorname{det}\left(d X\left(u_{j}\right)^{*} d X\left(u_{j}\right)\right)\right]^{1 / 2} \mid X\left(u_{j}\right)=0, j=1, \ldots, m\right]
$$

and $\operatorname{Den}_{X\left(u_{1}\right), \ldots, X\left(u_{m}\right)}$ is the density of $\left(X\left(u_{j}\right)\right)_{j=1}^{m}$.

If $k=n$, then equation (25) holds for any Borel set $B \subseteq U$ with the left hand side replaced by the factorial moment:

$$
\mathbb{E}\left[\prod_{j=1}^{m}\left[\mathcal{H}^{n-k}(\{X=0\} \cap B)-j+1\right]\right] .
$$

Remark 10. The equality (25) is valid even if one side of it (and hence the other) is infinite. Moreover, let $W: \mathbb{R}^{\alpha} \rightarrow \mathbb{R}^{\beta}$ be a continuous Gaussian field such that $(X, W)$ is Gaussian and suppose $f: \mathbb{R}^{n} \times C^{0}\left(\mathbb{R}^{\alpha}, \mathbb{R}^{\beta}\right) \rightarrow \mathbb{R}$ is a positive measurable function that is continuous when $C^{0}\left(\mathbb{R}^{\alpha}, \mathbb{R}^{\beta}\right)$ is equipped with with topology of uniform convergence 
on compact sets. Then, the formula (25) is valid with $\mathcal{H}^{n-k}(\{X=0\} \cap B)$ replaced by

$$
\left.\int_{\{X=0\} \cap B} f(u, W(u)) d \mathcal{H}^{n-k}\right|_{\{X=0\}}(u),
$$

and $Y_{m, X}\left(u_{1}, \ldots, u_{m}\right)$ replaced by $Y_{m, X, f}\left(u_{1}, \ldots, u_{m}\right)$, defined as

$$
\mathbb{E}\left[\prod_{j=1}^{m} f\left(u_{j}, W\left(u_{j}\right)\right)\left[\operatorname{det}\left(d X\left(u_{j}\right)^{*} d X\left(u_{j}\right)\right)\right]^{1 / 2} \mid X\left(u_{j}\right)=0, j=1, \ldots, m\right] .
$$

This statement when $f$ is bounded is a special case of [1, Thm. 6.10]. It can be extended to positive $f$ by considering the truncations $f_{N}:=\max (f, N), N \in \mathbb{N}$, and using the monotone convergence theorem.

2.1. Kac-Rice Hypotheses for Zeros. In this section, we prove that $\phi_{\infty}$ satisfies the hypotheses of the Kac-Rice Theorem. Since $\phi_{\infty}$ is almost surely smooth, Hypothesis (1) is satisfied. Hypothesis (2) requires that the distribution of

$$
\operatorname{ev}\left(\phi_{\infty} ; u_{1}, \ldots, u_{n}\right):=\left(\phi_{\infty}\left(u_{1}\right), \ldots, \phi_{\infty}\left(u_{m}\right)\right)
$$

be non-degenerate. Note that we have the following equality in law on the space of continuous functions:

$$
\phi_{\infty}(u) \stackrel{d}{=} \int_{S^{n-1}} \cos (\langle u, \omega\rangle)\left(\sum_{j=1}^{\infty} a_{j} \psi_{j}(\omega)\right) d \omega,
$$

where $\sum_{j=1}^{\infty} a_{j} \psi_{j}(\omega)$ is a white noise based in $L^{2}\left(S^{n-1}, \mathbb{R}\right)$ (i.e. $a_{j}$ are i.i.d. standard Gaussians and $\left\{\psi_{j}\right\}$ is an orthonormal basis for $\left.L^{2}\left(S^{n-1}, \mathbb{R}\right)\right)$. Let us write

$$
V:=\mathcal{F}^{-1}\left(L^{2}\left(S^{n-1}, \mathbb{R}\right)\right)=\left\{f(u)=\int_{S^{n-1}} \cos (\langle\omega, u\rangle) g(\omega) d \omega: g \in L^{2}\left(S^{n-1}, \mathbb{R}\right)\right\}
$$

for the real-valued functions on $\mathbb{R}^{n}$ with frequency 1 . Since ev $\left(\cdot ; u_{1}, \ldots, u_{m}\right)$ is linear and the law of $\phi_{\infty}$ is a non-degenerate Gaussian measure on $V$, it is enough to show that $\mathrm{ev}\left(\cdot ; u_{1}, \ldots, u_{m}\right)$ is surjective as a function on $V$ for every fixed collection of $m \geq 1$ distinct points $\left\{u_{\ell}\right\}_{\ell=1}^{m}$. The surjectivity of the linear functional $\phi \mapsto \operatorname{ev}\left(\phi ; u_{1}, \ldots, u_{m}\right)$ is equivalent to the linear independence of its components

$$
\phi \mapsto \phi\left(u_{\ell}\right)=\int_{S^{n-1}} \cos \left(\left\langle u_{\ell}, \omega\right\rangle\right) \widehat{\phi}(\omega) d \omega .
$$

Since $L^{2}\left(S^{n-1}, \mathbb{R}\right)$ separates points, this is implied by taking the real part of the following result.

Proposition 4 (Non-degeneracy for zero sets). Fix $n \geq 2$ and $m \geq 1$. Let $u_{1}, \ldots, u_{m} \in$ $\mathbb{R}^{n}$ be distinct. Then, the functions

$$
\left\{e^{i\left\langle u_{\ell}, \omega\right\rangle}: 1 \leq \ell \leq m\right\}
$$

are linearly independent on $S^{n-1}$. 
Proof. Suppose

$$
\sum_{\ell=1}^{m} a_{\ell} e^{i\left\langle u_{\ell}, \omega\right\rangle} \equiv 0, \quad a_{\ell} \in \mathbb{R}
$$

By multiplying by $e^{-i\langle u, \omega\rangle}$ for an appropriate $u \in \mathbb{R}^{n}$ we may assume that the values $\left|u_{\ell}\right|$ are positive and distinct. Recall the plane wave expansion (see e.g. [6, Thm. 2])

$$
e^{i\langle u, \omega\rangle}=\sum_{k=0}^{\infty} C_{k}\left(\frac{i|u|}{2}\right)^{k} j_{k+\alpha}(|u|) Z_{k}(\widehat{u}, \omega),
$$

where

$$
\alpha=\frac{n-2}{2}, \quad C_{k}=c_{k} \cdot d_{k}, \quad c_{k}=\frac{\Gamma(\alpha+1)}{\Gamma(\alpha+k+1)}, \quad \widehat{u}=\frac{u}{|u|},
$$

$d_{k}$ is the dimension of the space of spherical harmonics of degree $k$, the functions $j_{\nu}$ are normalized Bessel functions

$$
j_{\nu}(t)=\Gamma(\nu+1)\left(\frac{t}{2}\right)^{-\nu} J_{\nu}(t)
$$

solving $y^{\prime \prime}+\frac{2 \nu+1}{t} y^{\prime}+y=0$ with $y(0)=1$, and $Z_{k}(\widehat{u}, \omega)$ are the zonal harmonics of degree $k$ normalized by $\left\|Z_{k}(\widehat{u}, \cdot)\right\|_{L^{\infty}}=1$ for each $\widehat{u}, k$. The normalization of $Z_{k}$ implies

$$
\int_{S^{n-1}} Z_{k}\left(\omega_{0}, \omega\right) Z_{k}\left(\omega_{1}, \omega\right) d \omega=d_{k}^{-1} Z_{k}\left(\omega_{0}, \omega_{1}\right)
$$

for all $\omega_{0}, \omega_{1} \in S^{n-1}$. Substituting (30) into (29), we have

$$
\sum_{\ell=1}^{m} \sum_{k=0}^{\infty} C_{k} a_{\ell}\left(\frac{i\left|u_{\ell}\right|}{2}\right)^{k} j_{k+\alpha}\left(\left|u_{\ell}\right|\right) Z_{k}\left(\widehat{u}_{\ell}, \omega\right) \equiv 0 .
$$

For each $\widehat{y} \in S^{n-1}$ and $k \geq 0$ we integrate against $Z_{k}(\widehat{y}, \omega)$ to find

$$
\sum_{\ell=1}^{m} a_{\ell} c_{k}\left(\frac{i\left|u_{\ell}\right|}{2}\right)^{k} j_{k+\alpha}\left(\left|u_{\ell}\right|\right) Z_{k}\left(\widehat{u_{\ell}}, \widehat{y}\right)=0, \quad \forall k \geq 0, \quad \widehat{y} \in S^{n-1} .
$$

Let $\ell^{*}=\operatorname{argmax}\left\{\left|u_{\ell}\right|: 1 \leq \ell \leq m\right\}$, and recall that for $t \geq 0$ fixed

$$
j_{\nu}(t)=1+o(1), \quad \text { as } \nu \rightarrow \infty .
$$

Keeping in mind the normalization $\left\|Z_{k}(\widehat{u}, \cdot)\right\|_{L^{\infty}}=Z_{k}(\widehat{u}, \widehat{u})=1$, we divide (31) by $c_{k}\left(i\left|u_{\ell^{*}}\right| / 2\right)^{k}$, set $\widehat{y}=\widehat{u}_{\ell^{*}}$, use (32), and send $k \rightarrow \infty$ to conclude $a_{\ell^{*}}=0$. Repeating this for the $m-1$ remaining points completes the proof.

It remains to check that $\phi_{\infty}$ satisfies Hypothesis (3) in Theorem 2 . Since the law of $\phi_{\infty}$ is translation-invariant, it is enough to show that $\left(\phi_{\infty}(0), \partial_{1} \phi_{\infty}(0), \ldots, \partial_{n} \phi_{\infty}(0)\right)$ is a non-degenerate Gaussian vector. Just as with the discussion before Proposition 4 , but using the maps $\phi \mapsto \partial_{i} \phi(0)=\int_{S^{n-1}} \omega_{i} \widehat{\phi}(\omega) d \omega$, this is equivalent to the statement that the restrictions $\left\{1, \omega_{1}, \ldots, \omega_{n}\right\}$ of 1 and the $n$ coordinate functions are linearly independent functions on $S^{n-1}$. This is true since the zero set of an affine function is affine, and hence if it contains the unit sphere, then it must be $\mathbb{R}^{n}$. 
2.2. Kac-Rice Hypotheses for Critical Points. We continue to write $\phi_{\infty}$ for a frequency 1 random wave on $\mathbb{R}^{n}$. The purpose of this section is to check that the hypotheses of the Kac-Rice formula are satisfied by the Gaussian field $d \phi_{\infty}=$ $\left(\partial_{1} \phi_{\infty}, \ldots, \partial_{n} \phi_{\infty}\right)$. As in Section 2.1, $d \phi_{\infty}$ is almost surely smooth and hence satisfies Hypothesis (1). Also, since $d \phi_{\infty}$ takes values in $\mathbb{R}^{n}$, we do not need to check Hypothesis (3) in the statement of the Kac-Rice theorem. It therefore remains to check Hypothesis (2). We must show that for any distinct $u_{1}, \ldots, u_{m} \in \mathbb{R}^{n}$ the vector $\left(\partial_{i} \phi_{\infty}\left(u_{\ell}\right)\right)_{1 \leq i \leq n, 1 \leq \ell \leq m}$ has a non-degenerate distribution. By the same reasoning as preceded Proposition 4 , but using the maps

$$
\phi \mapsto \partial_{i} \phi\left(u_{\ell}\right)=\int_{S^{n-1}} \omega_{i} \sin \left(\left\langle u_{\ell}, \omega\right\rangle\right) \widehat{\phi}(\omega) d \omega
$$

instead of (28), the non-degeneracy of $\left(\partial_{i} \phi_{\infty}\left(u_{\ell}\right)\right)_{1 \leq i \leq n, 1 \leq \ell \leq m}$ is implied by the following result.

Proposition 5 (Non-degeneracy for critical sets). Let $u_{1}, \ldots, u_{m} \in \mathbb{R}^{n}$ be $m$ distinct points. Then, the functions

$$
\left\{\omega_{k} e^{i\left\langle u_{\ell}, \omega\right\rangle}: 1 \leq \ell \leq m, 1 \leq k \leq n\right\}
$$

are linearly independent on $S^{n-1}$.

Proof. Suppose

$$
\sum_{\ell=1}^{m} \sum_{k=1}^{n} a_{\ell, k} \omega_{k} e^{i\left\langle u_{\ell}, \omega\right\rangle} \equiv 0, \quad \omega \in S^{n-1}
$$

with $\overrightarrow{a_{\ell}}:=\left(a_{\ell, k}\right)_{k=1}^{n}$ not all zero. We begin by considering $n \geq 3$. In this case, we use that the degree $k$ zonal harmonic $Z_{k}(\omega, \cdot)$ is highly peaked at $\omega$ (see (39)). After mutiplying (33) by $e^{i\langle u, \omega\rangle}$ for an appropriately chosen $u$, we may assume

$$
\vec{a}_{\ell} \neq 0 \quad \Rightarrow \quad\left\langle\vec{a}_{\ell}, u_{\ell}\right\rangle \neq 0, \quad \forall \ell
$$

and that the points $\widehat{u}_{\ell}=u_{\ell} /\left|u_{\ell}\right| \in S^{n-1}$ are not antipodal and are distinct. Let $Q_{d}$ be a degree $d \neq 0$ harmonic homogeneous polynomial on $\mathbb{R}^{n}$. We have

$$
\sum_{k=1}^{n} \sum_{\ell=1}^{m} a_{\ell, k} \int_{S^{n-1}} e^{i\left\langle u_{\ell}, \omega\right\rangle} \omega_{k} Q_{d}(\omega) d \omega=0
$$

If $P$ is a homogeneous polynomial on $\mathbb{R}^{n}$ with degree $D$, then (see [6, Thm. 3$]$ )

$$
\int_{S^{n-1}} e^{i\langle u, \eta\rangle} P(\eta) d \eta=\sum_{j=0}^{[D / 2]} \kappa_{j, D} j_{\alpha+D-j}(|u|)\left(\Delta^{j} P\right)(u),
$$

where $\kappa_{j, D}=\left(\frac{i}{2}\right)^{D} \frac{(-1)^{j} \Gamma(\alpha+1)}{j ! \Gamma(\alpha+D+1-j)}$ and $j_{\nu}$ are normalized Bessel functions (see (30) ). Note that

$$
\Delta^{j}\left(x_{k} Q_{d}(x)\right)= \begin{cases}x_{k} Q_{d}(x) & j=0 \\ \partial_{x_{k}} Q_{d}(x) & j=1 \\ 0 & \text { otherwise }\end{cases}
$$


Hence, plugging (36) into ( $\left(\underline{35)}\right.$ ), with $P=\omega_{k} Q_{d}$ and $D=d+1$, we find that

$$
\sum_{k=1}^{n} \sum_{\ell=1}^{m} a_{\ell, k}\left(\frac{j_{\alpha+d+1}\left(\left|u_{\ell}\right|\right)}{\Gamma(\alpha+d+2)} u_{\ell, k} Q_{d}\left(u_{\ell}\right)-\frac{j_{\alpha+d}\left(\left|u_{\ell}\right|\right)}{\Gamma(\alpha+d+1)} \partial_{x_{k}} Q_{d}\left(u_{\ell}\right)\right)=0 .
$$

Note that, since $Q_{d}$ is homogeneous of degree $d$,

$$
\partial_{x_{k}} Q_{d}(x)=x_{k} d|x|^{d-2} Q_{d}(\widehat{x})+|x|^{d} \nabla_{\pi_{\widehat{x}}\left(e_{k}\right)}^{S^{n-1}} Q_{d}(\widehat{x}),
$$

where $e_{k}$ is the $k$-th unit vector, $\widehat{x}=x /|x|$ and $\pi_{\omega}(\vec{v})$ denotes the projection of the vector $\vec{v}$ onto the tangent fiber $T_{\omega} S^{n-1}$, for $\omega \in S^{n-1}$. Hence,

$$
\sum_{k=1}^{n} a_{\ell, k} \partial_{x_{k}} Q_{d}\left(u_{\ell}\right)=d\left|u_{\ell}\right|^{d-2} Q_{d}\left(\widehat{u}_{\ell}\right)\left\langle\overrightarrow{a_{\ell}}, u_{\ell}\right\rangle+\left|u_{\ell}\right|^{d} \nabla_{\pi_{\widehat{u}_{\ell}}\left(\overrightarrow{a_{\ell}}\right)}^{S^{n-1}} Q_{d}\left(\widehat{u}_{\ell}\right) .
$$

Substituting this into (37) and setting

$$
f_{d}(x):=|x|^{2} j_{\alpha+d+1}(|x|) \frac{\Gamma(\alpha+d+1)}{d \Gamma(\alpha+d+2)}-j_{\alpha+d}(|x|),
$$

we obtain

$$
\sum_{\ell=1}^{m}\left[f_{d}\left(u_{\ell}\right)\left\langle\overrightarrow{a_{\ell}}, u_{\ell}\right\rangle\left|u_{\ell}\right|^{d-2} Q_{d}\left(\widehat{u}_{\ell}\right)-\frac{j_{\alpha+d}\left(\left|u_{\ell}\right|\right)}{d}\left|u_{\ell}\right|^{d} \nabla_{\pi_{\widehat{u}_{\ell}}\left(\vec{a}_{\ell}\right)}^{S^{n-1}} Q_{d}\left(\widehat{u}_{\ell}\right)\right]=0 .
$$

Note that for $|x| \neq 0$, (32) implies that $f_{d}(x) \rightarrow-1$ as $d \rightarrow \infty$. Take $Q_{d}(\cdot)=Q_{d, \ell}(\cdot)=$ $Z_{d}\left(\widehat{u}_{\ell}, \cdot\right)$ to be the degree $d$ zonal harmonic centered at $\widehat{u}_{\ell}$. Note that since $n \geq 3$ and $\widehat{u}_{\ell}$ 's are not antipodal for different $\ell$,

$$
\lim _{d \rightarrow \infty} Q_{d, \ell}\left(\widehat{u}_{\ell^{\prime}}\right)=\delta_{\ell, \ell^{\prime}} \quad \text { and } \quad \lim _{d \rightarrow \infty} \nabla_{\pi_{\widehat{u}_{\ell}}\left(\vec{\alpha}_{\ell}\right)}^{S^{n-1}} Q_{d, \ell}\left(\widehat{u}_{\ell^{\prime}}\right)=0 \quad \forall \ell, \ell^{\prime} .
$$

Let $\ell^{*}=\operatorname{argmax}\left\{\left|u_{\ell}\right|: 1 \leq \ell \leq m\right\}$. Dividing (38) by $\left|u_{\ell^{*}}\right|^{d-2}$ and taking $d \rightarrow \infty$, we find $\left\langle\overrightarrow{a_{\ell^{*}}}, u_{\ell^{*}}\right\rangle=0$. Using (34), this shows $\overrightarrow{a_{\ell^{*}}}=0$. Repeating this argument for the $m-1$ remaining points completes the proof when $n \geq 3$.

If $n=2$ we cannot use the concentration of zonal harmonics. Instead, we argue by an explicit Fourier series computation. Suppose again that (33) holds. Write $\omega_{1}=\cos (\theta), \omega_{2}=\sin (\theta)$. After multiplying (33) by $e^{i\langle u, \omega\rangle}$, we may assume that the values $\left|u_{\ell}\right|$ are distinct and that $\left|u_{\ell}\right|>2$ for all $\ell$. We can also assume that, with $\widehat{u}_{\ell}=e^{i \theta_{\ell}}$, the arguments $\theta_{\ell}$ are not rational multiplies of $\pi$. Using the plane wave expansion (30), we have for $\theta \in[0,2 \pi]$ that

$$
F(\theta):=\sum_{\ell=1}^{m} \sum_{k=0}^{\infty}\left(\frac{i\left|u_{\ell}\right|}{2}\right)^{k} \frac{j_{k}\left(\left|u_{\ell}\right|\right)}{k !} \cos \left(k\left(\theta-\theta_{\ell}\right)\right)\left(a_{\ell, 1} \cos (\theta)+a_{\ell, 2} \sin (\theta)\right) \equiv 0 .
$$

We have used that $c_{k}=1 / k$ ! when $n=2$. By assumption, there exists a unique $\ell^{*}:=\operatorname{argmax}\left\{\left|u_{\ell}\right|: 1 \leq \ell \leq m\right\}$. Extracting the $N$-th Fourier coefficients in (40), multiplying them by $(N-1) ! \cdot\left(\frac{i\left|u_{\ell *}\right|}{2}\right)^{-N+1}$, and sending $N \rightarrow \infty$, we find that

$$
\lim _{N \rightarrow \infty}\left\langle\vec{a}_{\ell^{*}}, e^{i(N-1) \theta_{\ell^{*}}}\right\rangle=\lim _{N \rightarrow \infty}\left\langle\vec{a}_{\ell^{*}}^{\perp}, e^{i(N-1) \theta_{\ell^{*}}}\right\rangle=0
$$


where for any $v=\left(v_{1}, v_{2}\right)$ we set $v^{\perp}=\left(v_{2},-v_{1}\right)$. Hence, we must have that $\vec{a}_{\ell^{*}}=0$. Repeating this argument for the $m-1$ remaining points completes the proof.

2.3. Finiteness of the second moment for critical points. In this section we prove that if $R<\infty$ then

$$
\mathbb{E}\left[\left(\#\left\{d \phi_{\infty}(u)=0,|u| \leq R\right\}\right)^{2}\right]<\infty .
$$

Here, and in what follows, for a map $\psi: \mathbb{R}^{n} \rightarrow \mathbb{R}^{k}$ we write

$$
d \psi:=\left(\partial_{u_{1}} \psi, \ldots, \partial_{u_{n}} \psi\right) \quad \text { and } \quad \nabla_{\omega} d \psi=\left(\nabla_{\omega} \partial_{u_{1}} \psi, \ldots, \nabla_{\omega} \partial_{u_{n}} \psi\right),
$$

where $\nabla_{\omega}$ is the directional derivative in the direction of $\omega \in \mathbb{R}^{n}$. The results in Section 2.2 show that we may apply the Kac-Rice formula to the moments of the counting measure of $\left(d \phi_{\infty}\right)^{-1}(0)$. Hence, (41) is equivalent to showing

$$
Y_{2, d \phi_{\infty}}(u, v) \operatorname{Den}_{\left(d \phi_{\infty}(u), d \phi_{\infty}(v)\right)}(0,0) \in L_{\text {loc }}^{1}\left(\mathbb{R}^{n} \times \mathbb{R}^{n}\right),
$$

where $Y_{2, d \phi_{\infty}}$ is as in (25). Note that the density $\operatorname{Den}_{\left(d \phi_{\infty}(u), d \phi_{\infty}(v)\right)}(0,0)$ blows up at the diagonal $u=v$, so (42) is not immediate. Instead, it follows from Proposition 6. Proposition 7. Lemma 8, and the fact that $|u-v|^{2-n}$ is in $L_{\text {loc }}^{1}$. We state Propositions 6 and 7 below so that they can be applied to $\psi \in\left\{\phi_{\lambda}^{x}, d \phi_{\lambda}^{x}, \phi_{\infty}, d \phi_{\infty}\right\}$. In this section we only need the case $\psi=d \phi_{\infty}$, but in the proof of Theorems 1 1. 3 we will need the others as well.

Proposition 6. Fix $k=1, \ldots, n$. Suppose $\psi: B_{R} \rightarrow \mathbb{R}^{k}$ is a smooth centered Gaussian field, where $B_{R}$ is a ball of radius $R \in(0, \infty]$ in $\mathbb{R}^{n}$. Suppose that for every $u \in B_{R}$, the joint distribution of the vector $\left(\psi(u), \nabla_{\omega} \psi(u)\right)$ is uniformly non-degenerate:

$$
\inf _{u \in B_{R}} \inf _{\omega \in S^{n-1}} \operatorname{det} \operatorname{Cov}\left(\psi(u), \nabla_{\omega} \psi(u)\right)=c>0,
$$

where $\nabla_{\omega}$ is the directional derivative in the direction of $\omega$. Then, as $|u-v| \rightarrow 0$,

$$
\operatorname{Den}_{(\psi(u), \psi(v))}(0,0)=O\left(|u-v|^{-k}\right),
$$

where the implicit constant depends only on $c, n$, and $k$.

Proposition 7. Fix $k=1, \ldots, n$. Suppose $\psi: B_{R} \rightarrow \mathbb{R}^{k}, \quad \psi=\left(\psi_{\alpha}\right)_{\alpha=1}^{k}$, is a smooth centered Gaussian field, where $B_{R}$ is a ball of radius $R \in(0, \infty]$ in $\mathbb{R}^{n}$ satisfying the following assumptions:

(A1) $\forall u, v \in B_{R}, u \neq v$, the covariance matrix of $(\psi(v), \psi(u))$ is invertible:

$$
\left(\begin{array}{ll}
F(v, v) & F(v, u) \\
F(u, v) & F(u, u)
\end{array}\right)^{-1} \text { exists, }
$$

where $(F(u, v))_{\alpha, \beta}:=\operatorname{Cov}\left(\psi_{\alpha}(u), \psi_{\beta}(v)\right), \quad \alpha, \beta=1, \ldots, k$.

(A2) The smallest eigenvalue for the covariance matrix of $\psi(v)$ given $\psi(u)=0$ is bounded below by $|u-v|^{2}$. That is, $\exists c>0$ such that $\forall \xi \in \mathbb{R}^{k}$, with $\|\xi\|=1$,

$$
\xi^{T}\left(F(v, v)-F(v, u) F(u, u)^{-1} F(u, v)\right) \xi \geq c|u-v|^{2} .
$$

(A3) The entries of $F(u, v)$ are uniformly bounded in $C^{m}\left(B_{R} \times B_{R}\right)$ for all $m$. 
Define $Y_{2, \psi}(u, v)$ as in (25). Then, there exists $C>0$, depending only on $c$ and the $C^{3}$ norm of the entries of $F(u, v)$ on $B_{R} \times B_{R}$, so that, uniformly over $u \in B_{R}$, as $u \rightarrow v$,

$$
Y_{2, \psi}(u, v) \leq \begin{cases}C|u-v|^{2} & k=n, \\ C, & k<n .\end{cases}
$$

Lemma 8. The fields $\phi_{\infty}, d \phi_{\infty}$ satisfy the hypotheses of Propositions [6 and 7 with $R=\infty$.

Proof. The proofs for $\phi_{\infty}, d \phi_{\infty}$ are essentially the same, so we only provide details for the latter. The field $\phi_{\infty}$ is isotropic and translation invariant. Thus, $d \phi_{\infty}(u)$ is independent of $\nabla_{\omega} d \phi_{\infty}(u)$ for every $u, \omega$, and the distribution of both is independent of $u, \omega$. In particular, writing $u=\left(u_{1}, \ldots, u_{n}\right)$,

$\inf _{u \in B_{R}} \inf _{\omega \in S^{n-1}} \operatorname{det} \operatorname{Cov}\left(d \phi_{\infty}(u), \nabla_{\omega} d \phi_{\infty}(u)\right)=\operatorname{det}\left(\operatorname{Cov}\left(d \phi_{\infty}(0)\right)\right) \operatorname{det}\left(\operatorname{Cov}\left(\partial_{u_{1}} d \phi_{\infty}(0)\right)\right)$.

A direct computation using that $\int_{S^{n-1}} \omega_{i} \omega_{j} d \omega=\frac{1}{n} \delta_{i, j}$ shows that

$$
\operatorname{Cov}\left(d \phi_{\infty}(0)\right)=\frac{1}{n} \mathrm{Id}, \quad \text { and } \quad \operatorname{Cov}\left(\partial_{u_{1}} d \phi_{\infty}(0)\right)=L,
$$

where $L$ is the diagonal matrix

$$
L=\operatorname{Diag}\left(\kappa_{4}, \kappa_{2,2}, \ldots, \kappa_{2,2}\right), \quad \kappa_{4}:=\int_{S^{n-1}} \omega_{1}^{4} d \omega, \quad \kappa_{2,2}:=\int_{S^{n-1}} \omega_{1}^{2} \omega_{2}^{2} d \omega .
$$

Thus, $\phi_{\infty}$ satisfies the hypotheses of Proposition 6 . To check the hypotheses of Proposition 7, note that the invertibility of the covariance matrix of $\left(d \phi_{\infty}(v), d \phi_{\infty}(u)\right)$ is the content of Proposition 5, verifying (A1) (see also the paragraph before the statement of Proposition (5). Next, to check (A3), recall (10) and note that the $(\alpha, \beta)$ entry of $F(u, v)$ is

$$
(2 \pi)^{n / 2} \partial_{u_{\alpha}} \partial_{v_{\beta}}\left(J_{\frac{n-2}{2}}(|u-v|) /|u-v|^{\frac{n-2}{2}}\right) .
$$

This is a real analytic function that decays like $|u-v|^{-1 / 2}$ at infinity and hence has uniformly bounded derivatives of all orders for $u, v \in \mathbb{R}^{n}$. Finally, we check (A2) by a direct computation. Since $\phi_{\infty}$ is isotropic and stationary, we may assume $u=$ $\left(u_{1}, 0, \ldots, 0\right)$ and $v=\left(v_{1}, 0, \ldots, 0\right)$. For each $\alpha, \beta=1, \ldots, n$, Taylor expanding the exponential below we have

$$
\begin{aligned}
(F(u, v))_{\alpha, \beta} & =\partial_{u_{\alpha}} \partial_{u_{\beta}} \int_{S^{n-1}} e^{i(u-v) \omega} d \omega=\int_{S^{n-1}} e^{i(u-v) \omega_{\omega}} \omega_{\alpha} \omega_{\beta} d \omega \\
& =\int_{S^{n-1}} \omega_{\alpha} \omega_{\beta} d \omega-\int_{S^{n-1}}\left(u_{1}-v_{1}\right)^{2} \omega_{1}^{2} \omega_{\alpha} \omega_{\beta} d \omega+O\left(|u-v|^{4}\right) .
\end{aligned}
$$

Using that $\int_{S^{n-1}} \omega_{\alpha} \omega_{\beta} d \omega=\frac{1}{n} \delta_{\alpha, \beta}$ yields

$$
F(v, v)-F(u, v) F(u, v)^{T}=|u-v|^{2} 2 L\left(\operatorname{Id}+O\left(|u-v|^{2}\right)\right),
$$

where $L$ is as above. This completes the proof that (A2) holds and hence the proof of Lemma 8 as well. 
2.4. Proof of Proposition 6. Writing $\operatorname{Cov}_{\psi}(u, v):=\operatorname{Cov}(\psi(u), \psi(v))$, we have

$$
\operatorname{Den}_{(\psi(u), \psi(v))}(0,0)=(2 \pi)^{-n} \operatorname{det}\left(\operatorname{Cov}_{\psi}(u, v)\right)^{-1 / 2} .
$$

Proposition 6 is hence equivalent to proving that there exists $C$ such that for all $u \in \mathbb{R}^{n}$

$$
\frac{\sqrt{\operatorname{det} \operatorname{Cov}_{\psi}(u, v)}}{|u-v|^{k}} \geq C \quad \text { as } v \rightarrow u
$$

Write

$$
\psi(u)=\sum_{j=1}^{\infty} a_{j} \psi_{j}(u)=\langle\vec{a}, \Psi(u)\rangle_{\ell_{2}}, \quad \Psi(u)=\left(\Psi_{1}(u), \ldots, \Psi_{k}(u)\right)
$$

where $\Psi_{i}(u)=\left(\psi_{i, j}(u)\right)_{j=1}^{\infty}$ is a vector of smooth functions, whose existence is guaranteed by the Karhounen-Loeve Theorem. Note that

$$
\operatorname{Cov}_{\psi}(u, v)=\operatorname{Gram}\left(\Psi_{1}(u), \ldots, \Psi_{k}(u), \Psi_{1}(v), \ldots, \Psi_{k}(v)\right),
$$

where for any vectors $w_{i}$ in some inner product space $\operatorname{Gram}\left(w_{1}, \ldots, w_{\ell}\right)=\left(\left\langle w_{i}, w_{j}\right\rangle\right)_{1 \leq i, j \leq \ell}$ is the Gram matrix. By the Gram Identity

$$
\sqrt{\operatorname{det} \operatorname{Cov}_{\psi}(u, v)}=\|\Psi(u) \wedge \Psi(v)\|, \quad \Psi(u):=\Psi_{1}(u) \wedge \cdots \wedge \Psi_{k}(u)
$$

We have

$$
\frac{\sqrt{\operatorname{det} \operatorname{Cov}_{\psi}(u, v)}}{|u-v|^{k}}=\frac{\|\Psi(u) \wedge \Psi(v)\|}{|u-v|^{k}}=\left\|\Psi(u) \wedge \frac{\Psi(u)-\Psi(v)}{|u-v|^{k}}\right\|,
$$

where we have abbreviated $\Psi(u)-\Psi(v)=\wedge_{i=1}^{k}\left(\Psi_{i}(u)-\Psi_{i}(v)\right)$. Fix $u$ and suppose $v \rightarrow u$ through a sequence that achieves the liminf in (48). By passing to a further subsequence $\left\{v_{j}\right\}_{j}$ with $v_{j} \rightarrow u$, we may assume that there exists $w \in S^{n-1}$ so that

$$
w=\lim _{j \rightarrow \infty} \frac{u-v_{j}}{\left|u-v_{j}\right|} .
$$

Since $\psi$ is smooth, we therefore have

$$
\liminf _{v \rightarrow u} \frac{\sqrt{\operatorname{det}\left(\operatorname{Cov}_{\psi}(u, v)\right)}}{|u-v|^{k}}=\left\|\Psi(u) \wedge \nabla_{w} \Psi(u)\right\|=\sqrt{\operatorname{det} \operatorname{Cov}\left(\psi(u), \nabla_{w} \psi(u)\right)},
$$

where we have once again used the Gram Identity and have set $\nabla_{w} \Psi(u):=\bigwedge_{j=1}^{k} \nabla_{w} \Psi_{j}(u)$. The assumption (43) shows that the right hand since is uniformly bounded below over $u, \omega$, completing the proof.

2.5. Proof of Proposition 17. According to (25), $Y_{2, \psi}(u, v)$ has the form

$$
\mathbb{E}\left[\left(\operatorname{det}\left((d \psi(u))^{*} d \psi(u)\right) \operatorname{det}\left((d \psi(v))^{*} d \psi(v)\right)\right)^{1 / 2} \mid \psi(u)=\psi(v)=0\right] .
$$


Using that $2 a b \leq a^{2}+b^{2}$, it is sufficient to show that there exists $C>0$ so that

$$
\sup _{0<|u-v| \leq 1} \mathbb{E}\left[\operatorname{det}\left((d \psi(v))^{*} d \psi(v)\right) \mid \psi(u)=\psi(v)=0\right] \leq \begin{cases}C|u-v|^{2} & k=n, \\ C & k<n .\end{cases}
$$

Our proof of (50) relies on the following result.

Lemma 9. Suppose $\psi$ satisifies the hypotheses of Proposition 7 . Then,

$$
\sup _{\omega \in S^{n-1}} \sup _{0<|u-v| \leq 1} \sup _{t \in(0,1)} \operatorname{Cov}\left(\nabla_{\omega}^{2} \psi(t u+(1-t) v) \mid \psi(u)=\psi(v)=0\right)<\infty,
$$

where the bound applies to each entry of the covariance matrix and $\nabla_{\omega}$ is the directional derivative in the direction $\omega$. Similarly,

$$
\sup _{0<|u-v| \leq 1} \operatorname{Cov}(d \psi(v) \mid \psi(u)=\psi(v)=0)<\infty .
$$

We prove Lemma 9 in Section 2.6 by a direct computation. Before doing so, we show why it implies (50).

The case $k<n$, follows immediately by combining (52) and Lemma 10 below. To treat the slightly more technical case $k=n$, note that

$$
\operatorname{det}\left((d \psi(v))^{*} d \psi(v)\right)=\left\|\partial_{v_{1}} \psi(v) \wedge \cdots \wedge \partial_{v_{n}} \psi(v)\right\| .
$$

Write $w=\frac{u-v}{|u-v|}$. Note that since $\nabla_{\omega} \psi_{j}(v) \cdot \omega$ is the projection of $\nabla \psi_{j}(v)$ onto the direction of $\omega$, the $n$ vectors

$$
\vec{B}_{j}:=\nabla \psi_{j}(v)-\nabla_{\omega} \psi_{j}(v) \cdot \omega, \quad j=1, \ldots, n
$$

are contained in the orthogonal complement to $w$ in $\mathbb{R}^{n}$. Hence, there exist $a_{j} \in \mathbb{R}$, not all zero, so that

$$
\sum_{j=1}^{n} a_{j} \vec{B}_{j}=0 \quad \text { and } \quad \max _{j=1, \ldots, n}\left\{\left|a_{j}\right|\right\}=1 .
$$

Without loss of generality we will assume $a_{1} \neq 0$. Since wedge products are unchanged after adding to a column a linear combination of all columns, we therefore have

$$
\operatorname{det}\left((d \psi(v))^{*} d \psi(v)\right)=\left\|\sum_{j=1}^{n} a_{j} \nabla_{w} \psi_{j}(v) \cdot w \wedge d \psi_{2}(v) \wedge \cdots \wedge d \psi_{n}(v)\right\|^{2} .
$$

Since $\|\eta \wedge \zeta\| \leq\|\eta\|\|\zeta\|$, we conclude using again that $a b \leq a^{2}+b^{2}$ and CauchySchwarz, that the conditional expectation in (50) is bounded above by the product

$$
\begin{aligned}
& 2 \sum_{j=1}^{n} \mathbb{E}\left[\left|\nabla_{w} \psi_{j}(v)\right|^{4} \mid \psi(u)=\psi(v)=0\right]^{1 / 2} \\
& \times \quad \mathbb{E}\left[\left\|d \psi_{2}(v) \wedge \cdots \wedge d \psi_{n}(v)\right\|^{4} \mid \psi(u)=\psi(v)=0\right]^{1 / 2} .
\end{aligned}
$$

We will see that the first term is $O\left(|u-v|^{2}\right)$ and the second term is $O(1)$. To do that, we need the following fact. 
Lemma 10. Fix $N \geq 1$ and suppose $A$ is a symmetric positive semi-definite (PSD) $N \times N$ matrix. Denote by $\mu_{A}$ the associated centered Gaussian measure on $\mathbb{R}^{N}$. Then, for any measurable function $f: \mathbb{R}^{N} \rightarrow \mathbb{R}$ we have

$$
\int_{\mathbb{R}^{N}} f(x) d \mu_{A}(x)=\int_{\mathbb{R}^{N}} f\left(A^{1 / 2} x\right) d \mu_{\mathrm{Id}}(x) .
$$

In particular, denote by $\|A\|_{\infty}$ the magnitude of the largest entry in A. For each $d$, there exists a polynomial $p(t)$ of degree $d$ whose coefficients depend only on $d$ and $N$ so that

$$
|f(x)| \leq C(1+\|x\|)^{d} \forall x \in \mathbb{R}^{N} \Longrightarrow \int_{\mathbb{R}^{N}} f(x) d \mu_{A}(x) \leq C \cdot p\left(\|A\|_{\infty}\right) .
$$

Proof. Write $A^{1 / 2}$ for the PSD square root of $A$. If $x$ is distributed according to the standard Gaussian measure $\mu_{\mathrm{Id}}$ on $\mathbb{R}^{N}$, then $A^{1 / 2} x$ is distributed according to $\mu_{A}$. This proves (57). Relation (58) then follows from the fact that $\mu_{\text {Id }}$ has bounded moments of all orders.

We emphasize that Lemma 10 holds even when $A$ is not strictly positive definite. Note that relation (52), combined with (58) shows immediately that (56) is uniformly bounded over the set $0<|u-v| \leq 1$. To complete the proof of Proposition 7 we check that (51) implies that the expression in (55) is $O\left(|u-v|^{2}\right)$. To see this, note that if $d \psi(u)=d \psi(v)=0$, then the Mean Value Theorem yields, for each $j=1, \ldots, n$, the existence a point $c_{j}=c_{j}(\psi, u, v)$ in the interior of the line segment between $u$ and $v$ so that

$$
\nabla_{w} \psi_{j}\left(c_{j}\right)=0
$$

Hence, for each $j=1, \ldots, n$, we may write

$$
\begin{aligned}
\left|\nabla_{w} \psi_{j}(v)\right|^{4} & =\left|\int_{0}^{1}\right| v-c_{j}\left|\nabla_{\omega}^{2} \psi_{j}\left((1-t) c_{j}+t v\right) d t\right|^{4} \\
& \leq|u-v|^{4} \int_{0}^{1}\left|\nabla_{w}^{2} \psi_{j}((1-t) u+t v)\right|^{4} d t .
\end{aligned}
$$

Thus, using Jensen's inequality, we find that for all $u, v$ with $0<|u-v| \leq 1$, $\mathbb{E}\left[\left|\nabla_{\omega}^{2} \psi_{j}(v)\right|^{4} \mid \psi(u)=\psi(v)=0\right]$ is bounded above by

$$
|u-v|^{4} \sup _{t \in(0,1)} \mathbb{E}\left[\left|\nabla_{\omega}^{2} \psi_{j}((1-t) u+t v)\right|^{4} \mid \psi(u)=\psi(v)=0\right] .
$$

Combining (51) with (59), (60), and (58) therefore confirms (55) is bounded by a uniform constant times $|u-v|^{2}$, as claimed.

2.6. Proof of Lemma 9. The proofs of (52) and (51) are identical. We therefore give the details for (52) and omit the proof of (51). Fix $u \neq v$, and recall that $F(u, v)=$ $d_{u} d_{v} \mathbb{E}[\psi(u) \psi(v)]=\operatorname{Cov}(d \psi(u), d \psi(v))$. Note that a linear change of coordinates $(u, v) \mapsto(A u, A v)$ transforms $F(u, v)$ as follows:

$$
F(A u, A v)=A^{T} F(u, v) A .
$$


By Assumption (A1), the distribution of $d \psi(u)$ is non-degenerate. Hence, taking $A$ to be the square root of the inverse of the covariance matrix of this vector, we may work in coordinates so that

$$
F(u, u)=\mathrm{Id} .
$$

This is not really essential but will simplify the notation below. Assumption (A1) also guarantees that $(\psi(u), \psi(v))$ is non-degenerate and hence its covariance matrix is invertible. Thus,

$$
\begin{aligned}
\operatorname{Cov}\left(\left(d \psi_{1}(v), \ldots, d \psi_{k}(v)\right) \mid\right. & \psi(u)=\psi(v)=0) \\
& =\operatorname{Cov}\left(\left(d \psi_{1}(v), \ldots, d \psi_{k}(v)\right)\right)-R(u, v),
\end{aligned}
$$

where $R(u, v)$ is the $n k \times n k$ matrix

$$
R(u, v)=\left(\begin{array}{ccc}
\partial_{u_{1}} F(u, u) & \cdots & \partial_{u_{n}} F(u, u) \\
\partial_{u_{1}} F(u, v) & \cdots & \partial_{u_{n}} F(u, v)
\end{array}\right)^{T}\left(\begin{array}{cc}
X & Y \\
W & Z
\end{array}\right)\left(\begin{array}{ccc}
\partial_{u_{1}} F(u, u) & \cdots & \partial_{u_{n}} F(u, u) \\
\partial_{u_{1}} F(u, v) & \cdots & \partial_{u_{n}} F(u, v)
\end{array}\right)
$$

with

$$
\left(\begin{array}{cc}
X & Y \\
W & Z
\end{array}\right)=\left(\begin{array}{cc}
F(u, u) & F(u, v) \\
F(v, u) & F(v, v)
\end{array}\right)^{-1} .
$$

Assumption (A3) shows that it remains only to bound the entries of the matrix $R(u, v)$, which can be thought of as a collection of $k \times k$ blocks $(R(u, v))_{i j}$, with $i, j=1, \ldots, n$, given by

$\partial_{u_{i}} F(u, u)^{T}\left(X \partial_{u_{j}} F(u, u)+Y \partial_{u_{j}} F(u, v)\right)+\partial_{u_{i}} F(u, v)^{T}\left(W \partial_{u_{j}} F(u, u)+Z \partial_{u_{j}} F(u, v)\right)$.

Next, let $M_{\ell}=M_{\ell}(v)$ and $\varepsilon_{\ell}=\varepsilon_{\ell}(u, v)$ be $k \times k$ matrices so that after Taylor expansion at $u=v$ we have

$$
\partial_{u_{\ell}} F(u, v)=M_{\ell}+\varepsilon_{\ell}, \quad M_{\ell}=\left.\partial_{u_{\ell}} F(u, v)\right|_{u=v},
$$

for all $\ell=1, \ldots, n$. By Assumption (A3) the entries of $M_{\ell}(u)$ are uniformly bounded by a constant and the entries of $\varepsilon_{\ell}$ are uniformly bounded by a constant times $|u-v|$ :

$$
M_{\ell}=O(1), \quad \varepsilon_{\ell}(u, v)=O(|u-v|) .
$$

Then, for $i=1, \ldots, k, j=1, \ldots, n$,

$$
(R(u, v))_{i j}=M_{i}^{T}(X+Y+W+Z) M_{j}+M_{i}^{T}(Y+Z) \varepsilon_{j}+\varepsilon_{i}^{T}(W+Z) M_{j}+\varepsilon_{i}^{T} Z \varepsilon_{j} .
$$

Lemma 9 therefore reduces to showing that for every $i, j=1, \ldots, n$, we have

$$
(R(u, v))_{i j}=O(1) .
$$

To do this, note that using (61) and the usual block matrix inversion formula, we have

$$
\begin{array}{ll}
X=\mathrm{Id}+F(u, v) Z F(v, u), & W=-Z F(v, u), \\
Y=-F(u, v) Z, & Z=(F(v, v)-F(v, u) F(u, v))^{-1} .
\end{array}
$$

Using the expressions for $X, W$ it is straight forward to deduce that

$$
Y+Z=(\operatorname{Id}-F(u, v)) Z, \quad W+Z=Z(\operatorname{Id}-F(v, u))
$$

as well as

$$
X+Y+Z+W=\mathrm{Id}+(\operatorname{Id}-F(u, v)) Z(\mathrm{Id}-F(v, u)) .
$$


By Taylor expansion and Assumption (A3),

$$
\operatorname{Id}-F(u, v)=O(|u-v|), \quad \operatorname{Id}-F(v, u)=O(|u-v|),
$$

uniformly for $u, v \in B_{R}$. Assumption (A2) shows that for every $\xi \in \mathbb{R}^{n}$, we have

$$
\xi^{T} Z^{-1} \xi \geq c\|\xi\|^{2}|u-v|^{2} .
$$

Hence, the operator norm of $Z$ is bounded above by $c^{-1}|u-v|^{-2}$ and relation (64) follows by combining (63) with the explicit expression for $(R(u, v))_{i, j}$ just above (64). This completes the proof of (52).

\section{Local Results: Proof of Theorems 2 and 3}

We now prove Theorems 2 and 3 , Let $\phi_{\lambda} \in \operatorname{RW}_{\lambda}(M, g)$ and $x \in \mathcal{I} \mathcal{S}(M, g)$. Fix also a non-negative function $r_{\lambda}$ which satisfies $r_{\lambda}=o(\lambda)$ as $\lambda \rightarrow \infty$ and converges to a non-zero limit: $r_{\lambda} \rightarrow r_{\infty} \in(0, \infty]$.

We prove Theorem 2 for $r_{\infty}<\infty$ in Section 3.1. Theorem 3 for $r_{\infty}<\infty$ in Section 3.2, and Theorems 2 and 3 for $r_{\infty}<\infty$ in Section 3.3. Finally, in Section 3.4 we explicitly compute the corresponding constants.

3.1. Proof of Theorem 2 when $r_{\infty}<\infty$. We begin with the proof of Theorem 2 in the case $r_{\infty}<\infty$. We reproduce here the elegant argument communicated to us by G. Peccati. We seek to show that for any bounded measurable function $\psi: B\left(0, r_{\infty}\right) \rightarrow$ $\mathbb{R}$ we have

$$
\left\langle Z_{\lambda, r_{\lambda}}^{x}, \psi\right\rangle \stackrel{d}{\longrightarrow}\left\langle Z_{\infty, r_{\infty}}^{x}, \psi\right\rangle .
$$

To do this, we fix $R>r_{\infty}$ so that for all $\lambda \gg 1$ we have $r_{\lambda}<R$. Consider the sequence of laws on $C^{0}\left(B(0, R), \mathbb{R}^{n+1}\right)$, which we denote $\mu_{\lambda, R}^{x}$, associated to the random fields $J_{1}\left(\phi_{\lambda}^{x}\right)=\left(\phi_{\lambda}^{x}, \nabla \phi_{\lambda}^{x}\right)$ restricted to the compact set $B(0, R)$. By Kolmogorov's tightness criterion, this sequence is tight in $\lambda$ since the covariance functions of the fields are uniformly smooth and we have convergence of finite dimensional distributions (c.f. e.g. [20, Theorem 1.4.7]). Moreover, by the $C^{\infty}$ convergence of covariance functions (11), Prokhorov's theorem guarantees that $\mu_{\lambda, R}^{x}$ converges weakly to $\mu_{\infty, R}$. Hence, Skorohod's representation theorem gives a coupling of $J_{1}\left(\phi_{\lambda}^{x}\right)$ and $J_{1}\left(\phi_{\infty}^{x}\right)$ in which $J_{1}\left(\phi_{\lambda}^{x}\right)$ converges pointwise almost surely to $J_{1}\left(\phi_{\infty}^{x}\right)$. This implies (65). Indeed, with probability 1, by Bulinskaya's Lemma (cf eg Proposition 6.12 in [1] ) the zero set of $\phi_{\infty}^{x}$ inside of $B(0, R)$ is non-degenerate (i.e. a smooth co-dimension 1 submanifold). Thus, using that non-degenerate zero sets are stable under $C^{1}$ perturbations and dominated convergence yields the result.

This argument does not seem to hold for the case when $r_{\infty}=\infty$ since the domain $B(0, R)$ is non-compact and one cannot apply Kolmogorov's tightness criterion. Moreover, such an argument cannot possibly work for critical points of $\phi_{\lambda}^{x}$ since they are not stable under $C^{k}$ perturbations for any $k$ (see Theorem 3). 
3.2. Proof of Theorem 3 when $r_{\infty}<\infty$. We seek to apply the Kac-Rice formula for $m=1,2$ to conclude

$$
\mathbb{E}\left[\operatorname{Crit}_{\lambda, r_{\lambda}}(\psi)^{m}\right] \longrightarrow \mathbb{E}\left[\operatorname{Crit}_{\infty, r_{\infty}}(\psi)^{m}\right],
$$

as $\lambda \rightarrow \infty$. To do this, we begin by checking that the fields $d \phi_{\lambda}^{x}(u)$ satisfy the hypotheses of the Kac-Rice formula (25) for all $\lambda$ sufficiently large. Since the fields are smooth, we must only check the non-degeneracy condition (2). This condition is trivial when $m=1$. The following lemma verifies this for $m=2$.

Lemma 11. Let $r_{\lambda} \in(0, \infty)$ be a non-negative sequence with $\lim \inf r_{\lambda}>0$ and $r_{\lambda}=o(\lambda)$ both as $\lambda \rightarrow \infty$. Then, there exists $\Lambda$ so that for all $u \neq v$, and all $\lambda \geq \Lambda$, the distribution of $\left(d \phi_{\lambda}^{x}(u), d \phi_{\lambda}^{x}(v)\right)$ is non-degenerate. In particular, there exists $C, \Lambda>0$ so that for all $u \neq v$ with $u, v \in B_{r_{\lambda}}$

$$
\inf _{\lambda \geq \Lambda}\left[\operatorname{det} \operatorname{Cov}\left(d \phi_{\lambda}^{x}(u), d \phi_{\lambda}^{x}(v)\right)\right]^{1 / 2} \geq C|u-v|^{n} .
$$

Remark 11. We state Lemma 11 so that it applies both to the case $r_{\infty}<\infty$ and $r_{\infty}=\infty$. In this section we use only the case $r_{\infty}<\infty$, but in the next section we will apply it to $r_{\infty}=\infty$.

Proof of Lemma 11. We must check that $\phi_{\lambda}^{x}$ satisfies the hypothesis (43) of Proposition 6. That is, we seek to show that there exist $c>0$ and $\Lambda>0$ so that

$$
\inf _{\lambda \geq \Lambda} \inf _{u \in B_{r_{\lambda}}} \inf _{\omega \in S^{n-1}} \operatorname{det} \operatorname{Cov}\left(d \phi_{\infty}^{x}(u), \nabla_{\omega} d \phi_{\infty}^{x}(u)\right)=c>0 .
$$

The $C^{\infty}$ convergence of covarinace kernels (11) shows that

$$
\operatorname{det} \operatorname{Cov}\left(d \phi_{\lambda}^{x}(u), \nabla_{\omega} d \phi_{\lambda}^{x}(u)\right)=\operatorname{det} \operatorname{Cov}\left(d \phi_{\infty}^{x}(u), \nabla_{\omega} d \phi_{\infty}^{x}(u)\right)+o_{\lambda}(1),
$$

where the rate of convergence is uniform over $\omega \in S^{n-1}$ and $u \in B_{r_{\lambda}}$. By Lemma 8, the expression $\operatorname{det} \operatorname{Cov}\left(d \phi_{\infty}^{x}(u), \nabla_{\omega} d \phi_{\infty}^{x}(u)\right)$ is bounded from below away from 0 uniformly in $u$, completing the proof.

We have now verified that we can apply the Kac-Rice formula for finite $\lambda$ to study the convergence in (66) with $m=1,2$ (that we can apply it for $\lambda=\infty$ was already show in Proposition Section (5). It remains only to check that the limit of the expressions for finite $\lambda$ is the expression for $\lambda=\infty$. We will work out the details for the more complicated case $m=2$. Note that it is enough to check the case $\psi=\mathbf{1}_{A}$, the indicator of a bounded set $A$, since linear combinations of these are dense in the space of bounded measurable functions. For such $\psi$ we have, by the Kac-Rice formula that the factorial moment

$$
\frac{1}{\operatorname{vol}(B(0, R))^{2}} \mathbb{E}\left[\left(\sum_{\substack{d \phi_{\lambda}^{x}(u)=0 \\ u \in B(0, R)}} \psi(u)\right)\left(\sum_{\substack{d \phi_{\lambda}^{x}(u)=0 \\ u \in B(0, R)}} \psi(u)-1\right)\right]
$$

is equal to

$$
\frac{1}{\operatorname{vol}(B(0, R))^{2}} \int_{(A \cap B(0, R))^{2}} Y_{2, d \phi_{\lambda}^{x}}(u, v) \operatorname{Den}_{\left(d \phi_{\lambda}^{x}(u), d \phi_{\lambda}^{x}(v)\right)}(0,0) d u d v
$$

where

$$
Y_{2, d \phi_{\lambda}^{x}}(u, v)=\mathbb{E}\left[\left[\operatorname{det} \operatorname{Hess}\left(\phi_{\lambda}^{x}\right)(u)\right]^{\frac{1}{2}}\left[\operatorname{det} \operatorname{Hess}\left(\phi_{\lambda}^{x}\right)(v)\right]^{\frac{1}{2}} \mid d \phi_{\lambda}^{x}(u)=d \phi_{\lambda}^{x}(v)=0\right] .
$$


We prove that (68) converges, as $\lambda \rightarrow \infty$, to the analogous expression for $d \phi_{\infty}^{x}$ in two steps. First, we show that one can apply dominated covergence to the expression in (68). Then, we show that pointwise, when $u \neq v$, the limit of the integrand in (68) as $\lambda \rightarrow \infty$ is the integrand from the Kac-Rice formula applied to $d \phi_{\infty}$. In short, the proof of Theorem 3 when $r_{\infty}<\infty$ reduces to the following lemma.

Lemma 12. Let $r_{\lambda} \in(0, \infty)$ be any non-negative sequence with $\liminf r_{\lambda}>0$ and $r_{\lambda}=o(\lambda)$ both as $\lambda \rightarrow \infty$. There exist $C>0$ and $\Lambda>0$ so that for all $\lambda \geq \Lambda$ we have

$$
\sup _{0<|u-v| \leq r_{\lambda}} Y_{2, d \phi_{\lambda}^{x}}(u, v) \leq C|u-v|^{2}
$$

and

$$
\sup _{0<|u-v| \leq r_{\lambda}} \operatorname{Den}_{\left(d \phi_{\lambda}^{x}(u), d \phi_{\lambda}^{x}(v)\right)} \leq \frac{C}{|u-v|^{n}} .
$$

In particular, for all $\lambda \geq \Lambda Y_{2, d \phi_{\lambda}}(u, v) \operatorname{Den}_{\left(d \phi_{\lambda}^{x}(u), d \phi_{\lambda}^{x}(v)\right)}$ is dominated by $|u-v|^{-n+2}$, which is in $L^{1}$ with respect to $\operatorname{vol}\left(B_{r}\right)^{-2} \mathbf{1}_{\left\{u, v \in B_{r}\right\}} d u d v$ for all $r$. Moreover, for fixed $u \neq v$,

$$
\lim _{\lambda \rightarrow \infty} Y_{2, d \phi_{\lambda}}(u, v) \operatorname{Den}_{\left(d \phi_{\lambda}(u), d \phi_{\lambda}(v)\right)}(0,0)=Y_{2, d \phi_{\infty}}(u, v) \operatorname{Den}_{\left(d \phi_{\infty}(u), d \phi_{\infty}(v)\right)}(0,0) .
$$

Remark 12. We state Lemma 12 so that it applies both to the case $r_{\infty}<\infty$ and $r_{\infty}=\infty$. In this section we use only the case $r_{\infty}<\infty$, but in the next section we will apply it to $r_{\infty}=\infty$.

Proof. By Lemma 11, there exists $\Lambda_{0}>0$ so that for all $\lambda \in\left[\Lambda_{0}, \infty\right]$, and $u \neq v$ the random variable $\left(d \phi_{\lambda}^{x}(u), d \phi_{\lambda}^{x}(v)\right)$ is non-degenerate. Hence, in the notation of Lemma 11 .

$$
\operatorname{Den}_{\left(d \phi_{\lambda}^{x}(u), d \phi_{\lambda}^{x}(v)\right)}(0,0)=(2 \pi)^{-n}\left(\operatorname{det} \operatorname{Cov}_{d \phi_{\lambda}^{x}}(u, v)\right)^{-1 / 2} .
$$

Equation (70) now follows from (67). We now turn to showing (69) for which we need to verify that the field $d \phi_{\lambda}^{x}$ satisfies hypotheses (A1)-(A3) in Proposition 7 for all $\lambda$ sufficiently large. We already proved in Lemma 11] that assumption (A1) is satisfied for all $\lambda$ sufficiently large. Next, assumption (A3) holds by the uniform convergence of covariance kernels (11) and the fact that the limiting kernel $\Pi_{\infty}^{x}(u, v)$ for $\phi_{\infty}^{x}$ has uniformly bounded derivatives of all orders. To check (A2), we recall the notation $F(u, v)=d_{u} d_{v} \mathbb{E}\left[\phi_{\lambda}^{x}(u), \phi_{\lambda}^{x}(v)\right]$ from Proposition 7 with $\psi=d \phi_{\lambda}^{x}$. Note that for all $\xi \in \mathbb{R}^{n}$,

$$
\xi^{T}\left(F(v, v)-F(v, u) F(u, u)^{-1} F(u, v)\right) \xi \geq 0
$$

since $F(v, v)-F(v, u) F(u, u)^{-1} F(u, v)$ is the covariance matrix for $d \phi_{\lambda}^{x}(v)$ given that $d \phi_{\lambda}^{x}(u)$ vanishes. Thus, since the expression in the previous line vanishes at $u=v$, we may Taylor expand around $u=v$ to obtain

$$
\xi^{T}\left(F(v, v)-F(v, u) F(u, u)^{-1} F(u, v)\right) \xi=\xi^{T} M(u) \xi|u-v|^{2}+O\left(|u-v|^{3}\right),
$$

where $M$ is the second order term in the Talyor expansion of the matrix on the left hand side (and in particular is given by a finite number of derivatives of the covariance 
matrix $\Pi_{\lambda}^{x}(u, v)$ of $\phi_{\lambda}^{x}$ evaluated at $\left.u=v\right)$. Hence, by the computation of $L$ in Lemma 8, and the $C^{\infty}$ convergence of covariance kernels (11), we have

$$
\sup _{|u|<r_{\lambda}}\left|M(u)-2 \operatorname{Diag}\left(\kappa_{4}, \kappa_{2,2}, \ldots, \kappa_{2,2}\right)\right|=o_{\lambda}(1),
$$

with $\kappa_{4}=\int_{S^{n-1}} \omega_{1}^{4} d \omega, \kappa_{2,2}=\int_{S^{n-1}} \omega_{1}^{2} \omega_{2}^{2} d \omega$ as in Lemma 8. This proves (A2) and hence establishes (69). Finally, to prove (71), fix $u \neq v$. For all $\lambda$ sufficiently large, we have, by the $C^{\infty}$ convergence of covariance kernels (11), that

$$
\operatorname{Cov}_{d \phi_{\lambda}}(u, v)=\operatorname{Cov}_{d \phi_{\infty}}(u, v)+o_{\lambda}(1) .
$$

Moreover, $\operatorname{Cov}_{d \phi_{\lambda}}(u, v)$ is non-singular by Lemma 11 for all $\lambda$ sufficiently large. Therefore, by (72), we have

$$
\operatorname{Den}_{\left(d \phi_{\lambda}^{x}(u), d \phi_{\lambda}^{x}(v)\right)}=\operatorname{Den}_{\left(d \phi_{\infty}^{x}(u), d \phi_{\infty}^{x}(v)\right)}+o_{\lambda}(1) .
$$

Thus, it remains to show that as $\lambda \rightarrow \infty$

$$
Y_{2, d \phi_{\lambda}^{x}}(u, v)=Y_{2, d \phi_{\infty}^{x}}(u, v)+o_{\lambda}(1) .
$$

To do this, write $\Sigma_{u, v, \lambda}=\operatorname{Cov}\left(d^{2} \phi_{\lambda}(u), d^{2} \phi_{\lambda}(v) \mid d \phi_{\lambda}(u)=d \phi_{\lambda}(v)=0\right)$ for the covariance matrix of the second derivatives of $\phi_{\lambda}$ at $u, v$ given the first derivatives vanish. Note that for $u \neq v$ this covariance is well-defined since the distribution of $\left(d \phi_{\lambda}(u), d \phi_{\lambda}(v)\right)$ is non-degenerate. Therefore, using Lemma 10, we may write

$$
Y_{2, d \phi_{\lambda}^{x}}(u, v)=\int_{\mathbb{R}^{N}} f\left(\Sigma_{u, v, \lambda}^{1 / 2} \xi\right) d \mu_{\mathrm{Id}}(\xi),
$$

where $N=2 n(n+1)$ and $f$ is a function of polynomial growth. The entries of the covariance matrix $\Sigma_{u, v, \lambda}$, for our fixed $u, v$, are polynomials in $\Pi_{\lambda}^{x}(u, v)$ and its derivatives. Therefore,

$$
\Sigma_{u, v, \lambda}=\Sigma_{u, v, \infty}+o_{\lambda}(1) .
$$

The function $f$ has polynomial growth at infinity, and so we may apply dominated convergence to prove (173). This completes the proof of Lemma 12

3.3. Proofs of Theorems 2 and 3 when $r_{\infty}=\infty$. We begin by proving (14), for which it suffices to establish that $\lim _{\lambda \rightarrow \infty} \operatorname{Var}\left[Z_{\lambda, r_{\lambda}}^{x}(1)\right]=0$, which is precisely (15), together with the equality

$$
\lim _{\lambda \rightarrow \infty} \mathbb{E}\left[Z_{\lambda, r_{\lambda}}^{x}(1)\right]=\frac{1}{\sqrt{\pi n}} \frac{\Gamma\left(\frac{n+1}{2}\right)}{\Gamma\left(\frac{n}{2}\right)} .
$$

We prove (74) in Section 3.4 below and focus here on proving (15). To do this, note that we may apply the Kac-Rice formula (25) with $m=2$ to $\phi_{\lambda}^{x}$ for all $\lambda$ sufficiently large. Indeed, the fields $\phi_{\lambda}^{x}$ are smooth for all $\lambda$. Moreover, by the $C^{\infty}$ convergence of covariance kernels (11), we have

$$
\operatorname{Cov}\left(\phi_{\lambda}^{x}(u), d \phi_{\lambda}^{x}(u)\right)=\operatorname{Cov}\left(\phi_{\infty}^{x}(u), d \phi_{\infty}^{x}(u)\right)+o_{\lambda}(1) .
$$


Hence, since $\operatorname{Cov}\left(\phi_{\infty}^{x}(u), d \phi_{\infty}^{x}(u)\right)$ is non-degenerate by Proposition 4 , so is the left hand side for all $\lambda$ sufficiently large. Finally, again by (11), there exists $\Lambda>0$ so that

$$
\inf _{\lambda \geq \Lambda} \inf _{u \in B_{r_{\lambda}}} \inf _{\omega \in S^{n-1}} \operatorname{det} \operatorname{Cov}\left(\phi_{\lambda}^{x}(u), \nabla_{\omega} \phi_{\lambda}^{x}(v)\right)>0 .
$$

Hence, by Proposition 6 there exists $C>0$ so that

$$
\inf _{\substack{\lambda \geq \Lambda \\|u-v| \leq 1 \\ u \in B_{r_{\lambda}}}} \operatorname{Den}_{\left(\phi_{\lambda}^{x}(u), \phi_{\lambda}^{x}(v)\right)} \leq C|u-v|^{-1} .
$$

Thus, in particular, $\left.\operatorname{Den}_{\left(\phi_{\lambda}^{x}(u), \phi_{\lambda}^{x}(v)\right.}\right)$ exists for all $u \neq v$ and all $\lambda$ sufficiently large. We then have that, in the notation of the Kac-Rice formula, $\operatorname{Var}\left[Z_{\lambda, r_{\lambda}}^{x}(1)\right]$ is

$$
\begin{gathered}
\frac{1}{\operatorname{vol}\left(B_{r_{\lambda}}\right)^{2}} \int_{\mathbb{R}^{n}} \int_{\mathbb{R}^{n}} \mathbf{1}_{\left\{B_{r_{\lambda}} \times B_{r_{\lambda}}\right\}}(u, v)\left[Y_{2, \phi_{\lambda}^{x}}(u, v) \operatorname{Den}_{\phi_{\lambda}^{x}(u), \phi_{\lambda}^{x}(v)}(0,0)-\right. \\
\left.Y_{1, \phi_{\lambda}^{x}}(u) Y_{1, \phi_{\lambda}^{x}}(v) \operatorname{Den}_{\phi_{\lambda}^{x}(u)}(0) \operatorname{Den}_{\phi_{\lambda}^{x}(v)}(0)\right] d u d v .
\end{gathered}
$$

To show that (76) converges to zero, note that by (10) and (11), there exists $\Lambda>0$ so that, uniformly over $\lambda \geq \Lambda$ and $u, v \in B_{r_{\lambda}}$, for $\psi \in\left\{\phi_{\lambda}^{x}, \phi_{\infty}, d \phi_{\lambda}^{x}, d \phi_{\infty}\right\}$ we have

$$
\operatorname{Cov}(\psi(u), \psi(v))=\left(\begin{array}{cc}
\operatorname{Cov}(\psi(u)) & 0 \\
0 & \operatorname{Cov}(\psi(v))
\end{array}\right)+o(1)
$$

as $|u-v| \rightarrow \infty$. Hence, combining (77) with Lemma 10, we find

$$
\sup _{u, v \in B_{r_{\lambda}},|u-v| \geq r_{\lambda}^{1 / 2}}\left|Y_{2, \phi_{\lambda}^{x}}(u, v)-Y_{1, \phi_{\lambda}^{x}}(u) Y_{1, \phi_{\lambda}^{x}}(v)\right|=o(1) .
$$

Similarly,

$$
\sup _{u, v \in B_{r_{\lambda}},|u-v| \geq r_{\lambda}^{1 / 2}}\left|\operatorname{Den}_{\left(\phi_{\lambda}^{x}(u), \phi_{\lambda}^{x}(v)\right)}(0,0)-\operatorname{Den}_{\phi_{\lambda}^{x}(u)}(0) \operatorname{Den}_{\phi_{\lambda}^{x}(v)}(0)\right|=o(1)
$$

as $\lambda \rightarrow \infty$. On the other hand,

$$
\lim _{\lambda \rightarrow \infty} \frac{\operatorname{vol}\left\{(u, v) \in B_{r_{\lambda}} \times B_{r_{\lambda}}:|u-v| \geq r_{\lambda}^{1 / 2}\right\}}{\operatorname{vol}\left(B_{r_{\lambda}} \times B_{r_{\lambda}}\right)}=1 .
$$

Hence, the integrand in the expression above for $\operatorname{Var}\left[Z_{\lambda, r_{\lambda}}^{x}(1)\right]$ goes to zero pointwise on a sequence of sets whose measures tends to one. To complete the proof of (15) it therefore remains to show that the integrand in (76) is also uniformly dominated by an $L^{1}$ function. To do this, we repeat the proof of Lemma 12 to see that $\phi_{\lambda}$ satisfies the hypotheses of Proposition 7 for all $\lambda$ exceeding some $\Lambda>0$. Hence,

$$
\sup _{\lambda \geq \Lambda} \sup _{\substack{u \neq v \\ u \in B_{r_{\lambda}}}} Y_{2, \phi_{\lambda}}(u, v)<\infty .
$$

Similarly, simply by appealing to (10) and (11), we have

$$
\sup _{\lambda \geq \Lambda} \sup _{u \in B_{r_{\lambda}}} Y_{1, \phi_{\lambda}}(u) \operatorname{Den}_{\phi_{\lambda}^{x}(u)}(0)<\infty
$$


as well. These bounds, together with (75), yield that there is a constant $C>0$ so that the integrand in (76) is bounded by

$$
\frac{1}{\operatorname{vol}\left(B_{r_{\lambda}}\right)^{2}} \mathbf{1}_{\left\{B_{r_{\lambda}} \times B_{r_{\lambda}}\right\}}(u, v) \times \begin{cases}C|u-v|^{-1} & |u-v| \leq 1, \\ C & |u-v|>1 .\end{cases}
$$

This completes the proof of (14) and (15).

The proof of (17) and (18) is to repeat the preceeding argument with $\phi_{\lambda}$ replaced by $d \phi_{\lambda}$. The limit in (18) is independent of $x$ since $\operatorname{Crit}_{\infty, r}^{x}(1)$ is independent of linear changes of coordinates on $T_{x} M$ and, up to such a change of coordinates, $g_{x}$ coincides with the Euclidean metric. This completes the proof of Theorems 2 and 3 .

3.4. Computation of Explicit Constants. Before proving Theorem 1 we note that the statements in Remarks 4 and 8 follow from the extended Kac-Rice formula (Remark 10). We also note that the first moment asymptotics (14) follow from explicit computation of the limit in the Kac-Rice formula:

$$
\frac{1}{\sqrt{2 \pi}} \mathbb{E}\left[\left\|d \phi_{\infty}(0)\right\| \mid \phi_{\infty}(0)=0\right]=\left(\frac{n}{2 \pi}\right)^{n / 2} \int_{\mathbb{R}^{n}}|\xi| e^{-n|\xi|^{2} / 2} d \xi=\frac{1}{\sqrt{\pi n}} \frac{\Gamma\left(\frac{n+1}{2}\right)}{\Gamma\left(\frac{n}{2}\right)},
$$

which in particular confirms (74). To obtain (19), note that

$$
\operatorname{Cov}\left(\partial_{1} \phi_{\infty}(0), \partial_{2} \phi_{\infty}(0), \partial_{11}^{2} \phi_{\infty}(0), \partial_{22}^{2} \phi_{\infty}(0), \partial_{12}^{2} \phi_{\infty}(0)\right)
$$

is

$$
\left(\begin{array}{ccccc}
1 / 2 & 0 & 0 & 0 & 0 \\
0 & 1 / 2 & 0 & 0 & 0 \\
0 & 0 & 3 / 8 & 1 / 8 & 0 \\
0 & 0 & 1 / 8 & 3 / 8 & 0 \\
0 & 0 & 0 & 0 & 1 / 8
\end{array}\right)
$$

Hence,

$$
\begin{aligned}
& \mathbb{E}\left[\mid \text { det } \operatorname{Hess} \phi_{\infty}(0)|| d \phi_{\infty}(0)=0\right] \\
& =\frac{8}{(2 \pi)^{\frac{3}{2}}} \int_{\mathbb{R}^{3}}\left|x_{1} x_{2}-x_{3}^{2}\right| e^{-\frac{1}{2}\left(3 x_{1}^{2}+3 x_{2}^{2}-2 x_{1} x_{2}+8 x_{3}^{2}\right)} d x_{1} d x_{2} d x_{3} \\
& =\frac{1}{8(2 \pi)^{\frac{3}{2}}} \iiint_{\mathbb{R}^{3}}\left|2 y_{1}^{2}-y_{2}^{2}-y_{3}^{2}\right| e^{-\frac{1}{2}\left(y_{1}^{2}+y_{2}^{2}+y_{3}^{2}\right)} d y_{1} d y_{2} d y_{3} \\
& =\frac{1}{8(2 \pi)^{\frac{3}{2}}}\left[\int_{0}^{+\infty} r^{4} e^{-\frac{r^{2}}{2}} d r\right]\left[\int_{0}^{\pi} \int_{0}^{2 \pi}\left|3 \sin ^{2} \theta \cos ^{2} \varphi-1\right| \sin \theta d \varphi d \theta\right]=\frac{1}{4 \sqrt{6}} .
\end{aligned}
$$

The changes of variables we used are $y_{1}=x_{1}+x_{2}, y_{2}=\sqrt{2}\left(x_{1}-x_{2}\right), y_{3}=\sqrt{8} x_{3}$ and spherical coordinates $(r, \varphi, \theta) \in(0, \infty) \times(0,2 \pi) \times(0, \pi)$. Combining this with $\operatorname{Den}_{d \phi_{\infty}(0)}(0,0)=\frac{1}{\pi}$ and the Kac-Rice formula confirms (19). 


\section{Global Result - Proof of Theorem 1}

Let $\phi_{\lambda} \in \mathrm{RW}_{\lambda}(M, g)$ and suppose that $M$ is a manifold of isotropic scaling (Definition 11) and that random waves on $(M, g)$ have short-range correlations (Definition 2). We derive Theorem 1 from Theorems 2 and 3 , respectively. The derivation for zeros and critical points is essentially identical, so we will focus on proving Theorem 1 for critical points and will indicate the necessary changes to prove the zero sets statements as we go.

We first prove the estimates (3) and (44). It is enough to do this for indicator functions $\psi=\mathbf{1}_{A}$ for any $A \subseteq M$. Fix $\varepsilon>0$. For each $\lambda$ partition $A$ into finitely many disjoint subsets $\left\{U_{\alpha}\right\}_{\alpha \in S_{\lambda}}$ so that $A=\bigcup_{\alpha \in S_{\lambda}} U_{\alpha}$ and for some $c, C>0$

$$
c \cdot \lambda^{-1+\varepsilon} \leq \operatorname{diam}\left(U_{\alpha}\right) \leq C \cdot \lambda^{-1+\varepsilon}
$$

as $\lambda \rightarrow \infty$. For each $\alpha \in S_{\lambda}$ choose $x_{\alpha, \lambda} \in U_{\alpha}$ and write

$$
A_{\alpha, \lambda}:=\left\{u \in T_{x_{\alpha, \lambda}} M: \exp _{x_{\alpha, \lambda}}(u / \lambda) \in U_{\alpha} \cap A\right\} .
$$

We have,

$$
\begin{aligned}
\frac{\mathbb{E}\left[\operatorname{Crit}_{\lambda}\left(\mathbf{1}_{A}\right)\right]}{\lambda^{n} \operatorname{vol}(A)} & =\sum_{\alpha_{\lambda} \in S_{\lambda}} \frac{\operatorname{vol}\left(U_{\alpha}\right)}{\operatorname{vol}(A)} \mathbb{E}\left[\frac{\operatorname{Crit}_{\lambda}\left(\mathbf{1}_{U_{\alpha}}\right)}{\lambda^{n} \operatorname{vol}\left(U_{\alpha}\right)}\right] \\
& =\sum_{\alpha \in S_{\lambda}} \frac{\operatorname{vol}\left(U_{\alpha}\right)}{\operatorname{vol}(A)} \mathbb{E}\left[\operatorname{Crit}_{\lambda, \lambda_{\varepsilon}}^{x_{\alpha, \lambda}}\left(\mathbf{1}_{A_{\alpha, \lambda}}\right)\right],
\end{aligned}
$$

where Crit $x_{\lambda, r_{\lambda}}^{x}$ is defined in (16). Combining (18) (see Theorem 3) with Remarks 7 and 9 and the previous line proves (4).

We now seek to prove (5) and (6). As before, it is enough to take as our test function the indicator $\mathbf{1}_{A}$ for $A \subseteq M$ measurable. The proofs of (6) and (5) are identical, and we provide the details below for (6) .

For each $x \in M$ write $T_{x, \lambda}:=\operatorname{Var}\left(d \phi_{\lambda}(x)\right)=\left.d_{x} d_{y}\right|_{x=y} \Pi_{\lambda}(x, y)$. Proposition 5 and the discussion in Section 2.2 ensure that $T_{\lambda, x}$ is an invertible matrix at every $x$ for all $\lambda$ sufficiently large. We may therefore set

$$
\psi_{\lambda}(x):=T_{x, \lambda}^{-\frac{1}{2}} d \phi_{\lambda}(x)
$$

which yields

$$
\operatorname{Var}\left(\psi_{\lambda}(x)\right)=\mathrm{Id}, \quad \forall x \in M .
$$

Note that $\psi_{\lambda}$ and $d \phi_{\lambda}$ have the same zero set. It will turn out to be more convenient to study the variance for the size of the zero set of $\psi_{\lambda}$. Let us write

$$
X_{\lambda}:=\lambda^{-n} \#\left\{A \cap \psi_{\lambda}^{-1}(0)\right\} .
$$

We first check that we can apply the Kac-Rice formula to studying the first two moments of $X_{\lambda}$. Since $X_{\lambda}$ are smooth, we need only check that for $\lambda$ sufficiently large the Gaussian vector $\left(\psi_{\lambda}(x), \psi_{\lambda}(y)\right)$ is non-degenerate for all $x \neq y$. Note that for all $\lambda$ sufficiently large, $\psi_{\lambda}(x)$ is uniformly non-degenerate for all $x \in M$ by the isotropic scaling assumption (11). Thus, when $x, y \in M$ satisfy $d_{g}(x, y)>\lambda^{-\varepsilon}, \varepsilon \in(0,1)$, the short range correlation assumptions (2) and (77) immediately show that $\left(\psi_{\lambda}(x), \psi_{\lambda}(y)\right)$ 
is non-degenerate for all $\lambda$ large enough. In contrast, if $d_{g}(x, y)<\lambda^{-\varepsilon}$, we observe that $\operatorname{Cov}\left(\psi_{\lambda}(x), \psi_{\lambda}(y)\right)$ is

$$
P\left(\begin{array}{cc}
\left.d_{x} d_{y}\right|_{y=x} \Pi_{\lambda}(x, y) & d_{x} d_{y} \Pi_{\lambda}(x, y) \\
d_{x} d_{y} \Pi_{\lambda}(y, x) & \left.d_{x} d_{y}\right|_{x=y} \Pi_{\lambda}(x, y)
\end{array}\right) P, \quad P:=\left(\begin{array}{cc}
T_{x, \lambda}^{-1 / 2} & 0 \\
0 & T_{y, \lambda}^{-1 / 2}
\end{array}\right) .
$$

The middle matrix is precisely $\operatorname{Cov}\left(d \phi_{\lambda}(x), d \phi_{\lambda}(y)\right)$. We already saw in Lemma 11 that this matrix is invertible for every $x \neq y$ with $d_{g}(x, y)<\lambda^{-\varepsilon}$. Also note that $T_{x, \lambda}=\operatorname{Cov}\left(d \phi_{\lambda}(x)\right)=\lambda^{2} \operatorname{Cov}\left(d \phi_{\lambda}^{x}(0)\right)=\lambda^{2}\left(\operatorname{Cov}\left(d \phi_{\infty}(0)\right)+o(1)\right)$ uniformly for all $x \in M$. In particular,

$$
T_{x, \lambda}=\lambda^{2}\left(-\frac{1}{n} \operatorname{Id}+o_{\lambda}(1)\right) \quad \text { and } \quad T_{x, \lambda}^{-1 / 2}=\lambda^{-1}\left(-n^{1 / 2} \operatorname{Id}+o_{\lambda}(1)\right) .
$$

It then follows that $\operatorname{Cov}\left(\psi_{\lambda}(x), \psi_{\lambda}(y)\right)$ is also invertible for all $x \neq y$. This confirms that we can apply the Kac-Rice formula to write

$$
\operatorname{Var}\left[X_{\lambda}\right]=\iint_{A \times A}\left(I_{2, \psi_{\lambda}}(x, y)-I_{1, \psi_{\lambda}}(x) I_{1, \psi_{\lambda}}(y)\right) d v_{g}(x) d v_{g}(y),
$$

where

$$
I_{2, \psi_{\lambda}}(x, y)=Y_{2, \psi_{\lambda} / \lambda}(x, y) \cdot \operatorname{Den}_{\psi_{\lambda}(x) / \lambda, \psi_{\lambda}(y) / \lambda}(0,0)
$$

and

$$
I_{1, \psi_{\lambda}}(x)=Y_{1, \psi_{\lambda} / \lambda}(x) \cdot \operatorname{Den}_{\psi_{\lambda}(x) / \lambda}(0)
$$

with

$$
\begin{aligned}
& Y_{1, \psi_{\lambda} / \lambda}(x)=\mathbb{E}\left[\left\|D\left(\lambda^{-1} \psi_{\lambda}(x)\right)\right\| \mid \psi_{\lambda}(x)=0\right], \\
& Y_{2, \psi_{\lambda} / \lambda}(x, y)=\mathbb{E}\left[\left\|D\left(\lambda^{-1} \psi_{\lambda}(x)\right)\right\|\left\|D\left(\lambda^{-1} \psi_{\lambda}(y)\right)\right\| \mid \psi_{\lambda}(x)=\psi_{\lambda}(y)=0\right],
\end{aligned}
$$

where we have abbreviated $\|D f(x)\|=\left[\operatorname{det}\left(d f(x)^{*} d f(x)\right)\right]^{1 / 2}$. We will decompose the integral in (80) into three $\lambda$-dependent pieces using the following construction. There exist three positive numbers $C_{1}, C_{2}, C_{3}$ depending only on $n=\operatorname{dim}(M)$ with the following properties. For each $\varepsilon>0$ and every $\lambda>0$ there exists a collection of measurable sets $\left\{B_{\alpha}\right\}_{\alpha \in S_{\lambda, \varepsilon}}$ satisfying

i) $\# S_{\lambda, \varepsilon} \leq C_{1} \lambda^{n \varepsilon}$.

ii) $\operatorname{diam}\left(B_{\alpha}\right) \leq \lambda^{-\varepsilon}$ for every $\alpha \in S_{\varepsilon, \lambda}$.

iii) $\left\{x, y \in M: d_{g}(x, y)<\lambda^{-2 \varepsilon}\right\} \subset \bigcup_{\alpha \in S_{\lambda, \varepsilon}} B_{\alpha} \times B_{\alpha}$.

iv) For any $K>C_{2}$ and distinct $\alpha_{1}, \ldots, \alpha_{K} \in S_{\lambda, \varepsilon}$ we have $\bigcap_{i=1}^{K} B_{\alpha_{i}}=\emptyset$.

v) For each $B_{\alpha} \in S_{\lambda, \varepsilon}$ and every $2 \leq k \leq C_{2}$

$$
\text { \# }\left\{\text { distinct } \alpha_{2}, \ldots, \alpha_{k} \in S_{\lambda, \varepsilon} \mid B_{\alpha} \cap \bigcap_{i=2}^{k} B_{\alpha_{i}} \neq \emptyset\right\} \leq C_{3} \text {. }
$$

To see this, cover $M$ with finitely many coordinate charts. On each chart $g$ is uniformly comparable to the Euclidean metric. For the Euclidean metric, the existence of a collection satisfying (i)-(v) follows from standard covering arguments. Taking the 
union of these collections over the finite number of coordinate charts completes the construction of the sets $\left\{B_{\alpha}\right\}_{\alpha \in S_{\lambda, \varepsilon}}$ satisfying (i)-(v). Setting $\varepsilon:=\frac{n-1}{2 n}$, write

$$
\operatorname{Var}\left[X_{\lambda}\right]=\sum_{j=1}^{3} \underbrace{\iint_{\Omega_{j, \lambda} \cap A \times A}\left(I_{2, \psi_{\lambda}}(x, y)-I_{1, \psi_{\lambda}}(x) I_{1, \psi_{\lambda}}(y)\right) d v_{g}(x) d v_{g}(y)}_{=: W_{j, \lambda}},
$$

where

$$
\Omega_{1, \lambda}=\bigcup_{\alpha \in S_{\lambda, \varepsilon}} B_{\alpha} \times B_{\alpha}, \quad \Omega_{2, \lambda}=\Omega_{1, \lambda}^{c} \cap V_{\lambda}, \quad \Omega_{3, \lambda}=\Omega_{1, \lambda}^{c} \cap V_{\lambda}^{c},
$$

and

$$
V_{\lambda}=\left\{(x, y) \in M \times M: \max _{\alpha, \beta \in\{0,1\}}\left\{\lambda^{-\alpha-\beta}\left|\nabla_{x}^{\alpha} \nabla_{y}^{\beta} \operatorname{Cov}\left(\psi_{\lambda}(x), \psi_{\lambda}(y)\right)\right|\right\}>\lambda^{-\frac{n-1}{4}}\right\} .
$$

The proof of (6) now reduces to the following three estimates:

$$
W_{i, \lambda}=O\left(\lambda^{-\frac{n-1}{2}}\right), \quad \text { as } \quad \lambda \rightarrow \infty, \quad i=1,2,3 .
$$

We begin by proving (83) for $i=1$. Consider any $B \subseteq M$ with $\operatorname{diam}(B) \leq \operatorname{inj}(M, g)$ and fix $x \in B$. Write

$$
B_{x, \lambda}:=\left\{u \in T_{x} M: \exp _{x}(u / \lambda) \in B\right\},
$$

and note that for each $B \subset M$

$$
\frac{\#\left(\left\{\psi_{\lambda}^{x}=0\right\} \cap B_{x, \lambda}\right)}{\operatorname{vol}\left(B_{x, \lambda}\right)}=\frac{\#\left(\psi_{\lambda}^{-1}(0) \cap B\right)}{\lambda^{n} \operatorname{vol}(B)},
$$

where $\psi_{\lambda}^{x}(u)=\psi_{\lambda}\left(\exp _{x}(u / \lambda)\right)$. By the Kac-Rice formula,

$$
\begin{aligned}
\frac{1}{\operatorname{vol}(B)^{2}} \iint_{B \times B} & \left(I_{2, \psi_{\lambda}}(x, y)-I_{1, \psi_{\lambda}}(x) I_{1, \psi_{\lambda}}(y)\right) d v_{g}(x) d v_{g}(y) \\
= & \operatorname{Var}\left(\frac{\#\left(\psi_{\lambda}^{-1}(0) \cap B\right)}{\lambda^{n} \operatorname{vol}(B)}\right)=\operatorname{Var}\left(\frac{\#\left(\left\{\psi_{\lambda}^{x}=0\right\} \cap B_{x, \lambda}\right)}{\operatorname{vol}\left(B_{x, \lambda}\right)}\right) .
\end{aligned}
$$

Since $(M, g)$ is a manifold of isotropic scaling, this last expression is uniformly bounded over $x \in M$ (see Remark 9). Using the inclusion-exclusion formula and property (iv) of $S_{\lambda, \varepsilon}$, we have the following decomposition for the indicator function of $\Omega_{1, \lambda}$ :

$$
\mathbf{1}_{\Omega_{1, \lambda}}=\sum_{j=1}^{C_{2}}(-1)^{j+1} \sum_{\substack{\text { distinct } \alpha_{i} \in S_{\lambda, \varepsilon} \\ i=1, \ldots, j}} \mathbf{1}_{B_{\alpha_{1, \ldots, j}} \times B_{\alpha_{1, \ldots, j}}},
$$

where $B_{\alpha_{1, \ldots, j}}:=B_{\alpha_{1}} \cap \cdots \cap B_{\alpha_{j}}$. By properties (i) and (v), for each $j$, the number of terms in the inner sum is at most $C_{1} C_{3} \lambda^{n \varepsilon}$. Note that by (ii), we have $\operatorname{vol}(B) \leq \lambda^{-n \varepsilon}$ 
for each $B \in\left\{B_{\alpha}\right\}_{\alpha \in S_{\lambda, \varepsilon}}$. For each $\alpha \in S_{\lambda, \varepsilon}$, choose $x_{\alpha, \lambda} \in B_{\alpha}$. Relation (18) in Theorem 3 together with Remark 9 and (84) shows

$\sup \left\{\operatorname{Var}\left[\frac{\#\left(\psi_{\lambda}^{-1}(0) \cap B\right)}{\lambda^{n} \operatorname{vol}(B)}\right]: B \neq \emptyset\right.$ finite intersection of sets in $\left.\left\{B_{\alpha}\right\}_{\alpha \in S_{\lambda, \varepsilon}}\right\}=O(1)$

as $\lambda \rightarrow \infty$. Combining this with (84), we find

$W_{1, \lambda}=\sum_{j=1}^{C_{2}}(-1)^{j+1} \sum_{\substack{\text { distinct } \alpha_{i} \in S_{\lambda, \varepsilon} \\ i=1, \ldots, j}} \operatorname{vol}\left(B_{\alpha_{1, \ldots, j}}\right)^{2} \operatorname{Var}\left[\frac{\#\left(\psi_{\lambda}^{-1}(0) \cap B_{\alpha_{1, \ldots, j}}\right)}{\lambda^{n} \operatorname{vol}\left(B_{\alpha_{1, \ldots, j}}\right)}\right]=O\left(\lambda^{-n \varepsilon}\right)$,

which confirms (83) for $i=1$ since $\varepsilon=(n-1) /(2 n)$. Next, to prove (83) for $i=2,3$ we will need the following estimate:

$$
\| \lambda^{-|\alpha|-|\beta|} \nabla_{x}^{\alpha} \nabla_{y}^{\beta}\left(\operatorname{Cov}\left(\psi_{\lambda}(x), \psi_{\lambda}(y)\right)_{i, j} \|_{L^{2}(M \times M)}^{2}=O_{\alpha, \beta}\left(\lambda^{-n+1}\right)\right.
$$

as $\lambda \rightarrow \infty$ for all $\alpha, \beta \geq 0,1 \leq i, j \leq n$. We postpone the proof of (86) until the end of this section. Assuming it for the moment, abbreviate

$$
\operatorname{Cov}\left(\psi_{\lambda}(x), \psi_{\lambda}(y)\right)=\Sigma_{\lambda}(x, y)=\left(\begin{array}{cc}
\Sigma_{x, x} & \Sigma_{x, y} \\
\Sigma_{x, y}^{T} & \Sigma_{y, y}
\end{array}\right) .
$$

By construction

$$
\Sigma_{x, x}=\Sigma_{y, y}=\mathrm{Id},\left.\quad d_{x}\right|_{x=y} \Sigma_{x, y}=0 .
$$

Combining Chebyshev's inequality with the definition of $V_{\lambda}$ with (86) yields

$$
\operatorname{vol}_{g}\left(V_{\lambda}\right)=O\left(\lambda^{-\frac{n-1}{2}}\right) \text {. }
$$

Next, we claim that

$$
\sup _{\substack{x, y \in V_{\lambda} \\ d_{g}(x, y)>\lambda^{-2 \varepsilon}}}\left|I_{2, \psi_{\lambda}}(x, y)-I_{1, \psi_{\lambda}}(x) I_{1, \psi_{\lambda}}(y)\right|=O(1)
$$

as $\lambda \rightarrow \infty$. Indeed, the Definition 2 of short-range correlations ensures that the density factor $\operatorname{Den}_{\psi_{\lambda}(x), \psi_{\lambda}(y)}(0,0)$ is uniformly bounded above on $V_{\lambda} \cap\left\{d_{g}(x, y)>\lambda^{-2 \varepsilon}\right\}$. Combining Lemma [10] with Lemma 13 below, we find that $Y_{2, \psi_{\lambda}}(x, y)$ and $Y_{1, \psi_{\lambda}}(x) Y_{1, \psi_{\lambda}}(y)$ are uniformly bounded on $V_{\lambda} \cap\left\{d_{g}(x, y)>\lambda^{-2 \varepsilon}\right\}$. Let

$$
M_{\lambda}(x, y)=\operatorname{Cov}\left(\lambda^{-1} d \psi_{\lambda}(x), \lambda^{-1} d \psi_{\lambda}(y) \mid \psi_{\lambda}(x)=\psi_{\lambda}(y)=0\right) .
$$

Lemma 13. There exists $C>0$ such that

$$
\sup _{x \neq y} M_{\lambda}(x, y) \leq C,
$$

where the inequality applies to each entry of $M_{\lambda}(x, y)$.

Proof. The idea of the proof is that the estimate (91) in the far off-diagonal regime (when $d_{g}(x, y)>\lambda^{-\varepsilon}$ ) essentially follows immediately from the pointwise Weyl Law and the short range correlation assumption (2). In the near off-diagonal regime $0<$ $d_{g}(x, y)<\lambda^{-\varepsilon}$ the estimate (91) follows closely the proof of Lemma 9, which gives 
essentially the same result but for the local fields $d \phi_{\lambda}^{x_{0}}$. But, since $\psi_{\lambda}(x)=T_{x, \lambda}^{-1 / 2} d \phi_{\lambda}(x)$ and the matrices $T_{x, \lambda} \approx \lambda^{-2}$ Id (see (79) ), the two fields have the same local behavior. We now provide the details. Note that

$$
M_{\lambda}(x, y)=\lambda^{-2} \operatorname{Cov}\left(d \psi_{\lambda}(x), d \psi_{\lambda}(y)\right)-\lambda^{-2} A(x, y)^{T} \operatorname{Cov}\left(\psi_{\lambda}(x), \psi_{\lambda}(y)\right)^{-1} A(x, y),
$$

where

$$
A=\left(\begin{array}{ll}
\operatorname{Cov}\left(d \psi_{\lambda}(x), \psi_{\lambda}(x)\right) & \operatorname{Cov}\left(d \psi_{\lambda}(x), \psi_{\lambda}(y)\right) \\
\operatorname{Cov}\left(d \psi_{\lambda}(y), \psi_{\lambda}(x)\right) & \operatorname{Cov}\left(d \psi_{\lambda}(y), \psi_{\lambda}(y)\right)
\end{array}\right),
$$

and we will see below that for all $x \neq y$ the matrix $\operatorname{Cov}\left(\psi_{\lambda}(x), \psi_{\lambda}(y)\right)$ is invertible. Since $\operatorname{Cov}(A X, B Y)=A^{T} \operatorname{Cov}(X, Y) B$, the covariance matrix $\operatorname{Cov}\left(d \psi_{\lambda}(x), d \psi_{\lambda}(y)\right)$ is

$$
\left(\begin{array}{ll}
C(x, x) & C(x, y) \\
C(y, x) & C(y, y)
\end{array}\right), \quad C(x, y)=d_{x} d_{y}\left(T_{x, \lambda}^{-1 / 2} d_{x} d_{y} \Pi_{\lambda}(x, y) T_{y, \lambda}^{-1 / 2}\right) .
$$

Recall that for any $\varepsilon \in(0,1)$, the short range correlations assumption (2) gives that for any multi-indices $\alpha, \beta$

$$
\sup _{d_{g}(x, y)>\lambda^{-\varepsilon}}\left|d_{x}^{\alpha} d_{y}^{\beta} \Pi_{\lambda}(x, y)\right|=O\left(\lambda^{|\alpha|+|\beta|}\right) ;
$$

and, moreover, if $|\alpha|+|\beta|$ is odd, then

$$
\left|d_{x}^{\alpha} d_{y}^{\beta}\right|_{x=y} \Pi_{\lambda}(x, y) \mid=o\left(\lambda^{|\alpha|+|\beta|}\right) .
$$

Hence, $\lambda^{-2} \operatorname{Cov}\left(d \psi_{\lambda}(x), d \psi_{\lambda}(y)\right)$ is uniformly bounded for all $x, y \in M$, and it remains to check that the second term in (92) is also uniformly bounded for all $x \neq y$. We do this, by considering separately the near off-diagonal and far off-diagonal regimes separately. We start with far off-diagonal regime in which we fix $\varepsilon>0$ and consider points $x, y$ with $d_{g}(x, y)>\lambda^{-\varepsilon}$. Combining (78) with (79) yields

$$
\operatorname{Cov}\left(\psi_{\lambda}(x), \psi_{\lambda}(y)\right)^{-1}=\operatorname{Id}+o_{\lambda}(1) .
$$

Hence, there exists $C>0$ such that for all $\lambda$ sufficiently large

$$
\sup _{d_{g}(x, y)>\lambda^{-\varepsilon}} M_{\lambda}(x, y) \leq C .
$$

To handle the near off-diagonal regime, where $0<d_{g}(x, y)<\lambda^{-\varepsilon}$, let us write in local coordinates

$$
x=x_{0}+\frac{u}{\lambda}, \quad y=x_{0}+\frac{v}{\lambda}, \quad \psi_{\lambda}^{x_{0}}(u):=\psi_{\lambda}\left(x_{0}+\frac{u}{\lambda}\right) .
$$

Thus, with $X, Y, W, Z$ as in Lemma 9, the expression (78) becomes

$\operatorname{Cov}\left(\psi_{\lambda}(x), \psi_{\lambda}(y)\right)^{-1}=\left(\begin{array}{cc}\lambda^{-1} T_{x, \lambda}^{1 / 2} & 0 \\ 0 & \lambda^{-1} T_{y, \lambda}^{1 / 2}\end{array}\right)\left(\begin{array}{cc}X & Y \\ W & Z\end{array}\right)\left(\begin{array}{cc}\lambda^{-1} T_{x, \lambda}^{1 / 2} & 0 \\ 0 & \lambda^{-1} T_{y, \lambda}^{1 / 2}\end{array}\right)$,

where the extra factors of $\lambda$ come from the fact that $\lambda^{-1} d \psi_{\lambda}(x)=d \psi_{\lambda}^{x_{0}}(u)$. Thus, we find that the second term in (92) equals

$$
B^{T}\left(\begin{array}{cc}
X & Y \\
W & Z
\end{array}\right) B \quad \text { with } \quad B=\left(\begin{array}{cc}
M \lambda^{-1} T_{x, \lambda}^{1 / 2} & (M+\varepsilon) \lambda^{-1} T_{y, \lambda}^{1 / 2} \\
(M+\varepsilon) \lambda^{-1} T_{x, \lambda}^{1 / 2} & (M+\varepsilon) \lambda^{-1} T_{y, \lambda}^{1 / 2}
\end{array}\right),
$$


where $M=M(x)$ and $\varepsilon=\varepsilon(x, y)$ are matrices with $M=\mathbb{E}\left[\lambda^{-1} d_{x} \psi_{\lambda}(x) \psi_{\lambda}(x)\right]$ and $\varepsilon=O(|x-y|)$. The $2 \times 2$ block matrix resulting from multiplying the three terms in (95) is a function only of $X+Y+W+Z, \varepsilon^{T}(Y+Z),(W+Z) \varepsilon$ and $\varepsilon^{T} Z \varepsilon$ times the matrices $\lambda^{-1} T_{x, \lambda}^{1 / 2}$. The estimates in (79) combined with the bounds just below (64) show that each such term is uniformly bounded, completing the proof of Lemma 13 .

Combining (88) with (89), we have

$$
\int_{\Omega_{2, \lambda}}\left(I_{2, \psi_{\lambda}}(x, y)-I_{1, \psi_{\lambda}}(x) I_{1, \psi_{\lambda}}(y)\right) d v_{g}(x) d v_{g}(y)=O\left(\operatorname{vol}_{g}\left(V_{\lambda}\right)\right)=O\left(\lambda^{-\frac{n-1}{2}}\right),
$$

confirming (83) for $i=2$. Finally, we check (83) for $i=3$. By definition of $V_{\lambda}$,

$$
\Sigma_{\lambda}(x, y)=\mathrm{Id}+\left(\begin{array}{cc}
0 & O\left(\lambda^{-\frac{n-1}{4}}\right) \\
O\left(\lambda^{-\frac{n-1}{4}}\right) & 0
\end{array}\right),
$$

with the error terms uniform over $x, y \in V_{\lambda}^{c}$. Hence,

$$
\operatorname{Den}_{\psi_{\lambda}(x), \psi_{\lambda}(y)}(0,0)=\operatorname{det}\left(2 \pi \Sigma_{\lambda}(x, y)\right)^{-1 / 2}=\operatorname{Den}_{\psi_{\lambda}(x)}(0) \operatorname{Den}_{\psi_{\lambda}(y)}(0)+O\left(\lambda^{-\frac{n-1}{2}}\right) .
$$

Thus,

$$
W_{3, \lambda}=\int_{\Omega_{3, \lambda}}\left(Y_{2, \psi_{\lambda}}(x, y)-Y_{1, \psi_{\lambda}}(x) Y_{1, \psi_{\lambda}}(y)\right) d v_{g}(x) d v_{g}(y)+O\left(\lambda^{-\frac{n-1}{2}}\right),
$$

and proving (83) for $i=3$ reduces to showing

$$
\sup _{(x, y) \in \Omega_{3, \lambda}}\left|Y_{2, \psi_{\lambda}}(x, y)-Y_{1, \psi_{\lambda}}(x) Y_{1, \psi_{\lambda}}(y)\right|=O\left(\lambda^{-\frac{n-1}{2}}\right) .
$$

Write

$\operatorname{Cov}\left(\lambda^{-1} \psi_{\lambda}(x), \lambda^{-1} \psi_{\lambda}(y), \lambda^{-1} d \psi_{\lambda}(x), \lambda^{-1} d \psi_{\lambda}(y)\right)=\lambda^{-2}\left(\begin{array}{cc}\Sigma_{\lambda}(x, y) & \lambda B_{\lambda}(x, y) \\ \lambda B_{\lambda}(x, y)^{T} & \lambda^{2} C_{\lambda}(x, y)\end{array}\right)$, where by the definition of $V_{\lambda}$ we have

$$
\begin{aligned}
& B_{\lambda}(x, y)=\left(\begin{array}{cc}
0 & \lambda^{-1} d_{x} \Sigma_{\lambda}(x, y) \\
\lambda^{-1} d_{y} \Sigma_{\lambda}(x, y) & 0
\end{array}\right)=\left(\begin{array}{cc}
0 & O\left(\lambda^{-\frac{n-1}{4}}\right) \\
O\left(\lambda^{-\frac{n-1}{4}}\right) & 0
\end{array}\right) \\
& C_{\lambda}(x, y)=\left(\begin{array}{cc}
\left.\lambda^{-2} d_{x} d_{y}\right|_{x=y} \Sigma_{\lambda}(x, y) & \lambda^{-2} d_{x} d_{y} \Sigma_{\lambda}(x, y) \\
\lambda^{-2} d_{x} d_{y} \Sigma_{\lambda}(x, y) & \left.\lambda^{-2} d_{x} d_{y}\right|_{x=y} \Sigma_{\lambda}(x, y)
\end{array}\right)=\left(\begin{array}{cc}
C_{\lambda}(x) & O\left(\lambda^{-\frac{n-1}{4}}\right) \\
O\left(\lambda^{-\frac{n-1}{4}}\right) & C_{\lambda}(y)
\end{array}\right),
\end{aligned}
$$

where the error terms are uniform in $(x, y) \in \Omega_{3, \lambda}$ and there exists $C=C(\operatorname{dim}(M))>0$ so that

$$
\sup _{x \in M}\left|C_{\lambda}(x)-C \cdot \operatorname{Id}\right|=o(1)
$$

as $\lambda \rightarrow \infty$ by the poinwise Weyl law. Hence, with $M_{\lambda}(x, y)$ defined as in (90)

$$
M_{\lambda}(x, y)=\left(\begin{array}{cc}
C_{\lambda}(x) & 0 \\
0 & C_{\lambda}(y)
\end{array}\right)\left(\operatorname{Id}+\left(\begin{array}{ll}
O\left(\lambda^{-\frac{n-1}{2}}\right) & O\left(\lambda^{-\frac{n-1}{4}}\right) \\
O\left(\lambda^{-\frac{n-1}{4}}\right) & O\left(\lambda^{-\frac{n-1}{2}}\right)
\end{array}\right)\right) .
$$

In particular,

$$
\operatorname{det}\left(M_{\lambda}(x, y)\right)=\operatorname{det}\left(C_{\lambda}(x)\right) \operatorname{det}\left(C_{\lambda}(y)\right)\left(1+O\left(\lambda^{-\frac{n-1}{2}}\right)\right)
$$


and

$$
\left(M_{\lambda}(x, y)\right)^{-1}=\left(\begin{array}{cc}
C_{\lambda}(x)^{-1} & 0 \\
0 & C_{\lambda}(y)^{-1}
\end{array}\right)+\left(\begin{array}{ll}
Q_{\lambda}(x, y) & R_{\lambda}(x, y) \\
R_{\lambda}(y, x) & Q_{\lambda}(x, y)
\end{array}\right),
$$

where $R_{\lambda}(x, y), Q_{\lambda}(x, y)$ are matrices that satisfy the entrywise estimates

$$
\sup _{(x, y) \in \Omega_{3, \lambda}} R_{\lambda}(x, y)=O\left(\lambda^{-\frac{n-1}{4}}\right) \quad \text { and } \sup _{(x, y) \in \Omega_{3, \lambda}} Q_{\lambda}(x, y)=O\left(\lambda^{-\frac{n-1}{2}}\right) .
$$

Then, since $d \psi_{\lambda}(x)$ is uncorrelated from $\psi_{\lambda}(x)$ at each $x \in M$, we find using (98) that $Y_{2, \psi_{\lambda}}(x, y)-Y_{1, \psi_{\lambda}}(x) Y_{1, \psi_{\lambda}}(y)$ is

$$
\iint_{\mathbb{R}^{2 n}} \frac{|\xi||\zeta| e^{-\frac{1}{2}\left\langle C_{\lambda}(x)^{-1} \xi, \xi\right\rangle-\frac{1}{2}\left\langle C_{\lambda}(y)^{-1} \zeta, \zeta\right\rangle}}{(2 \pi)^{n}\left(\operatorname{det} C_{\lambda}(x) \operatorname{det} C_{\lambda}(y)\right)^{\frac{1}{2}}}\left(e^{-\left\langle R_{\lambda}(x, y) \zeta, \xi\right\rangle-\frac{1}{2}\left\langle Q_{\lambda}(x, y)\left(\begin{array}{c}
\xi \\
\zeta
\end{array}\right),\left(\begin{array}{l}
\xi \\
\zeta
\end{array}\right)\right\rangle}-1\right) d \xi d \zeta
$$

plus $O\left(\lambda^{-\frac{n-1}{2}}\right)$ where the implied constant is uniform over $(x, y) \in \Omega_{3, \lambda}$. Observe that

$$
e^{-\left\langle R_{\lambda}(x, y) \zeta, \xi\right\rangle-\frac{1}{2}\left\langle Q_{\lambda}(x, y)\left(\begin{array}{l}
\xi \\
\zeta
\end{array}\right),\left(\begin{array}{l}
\xi \\
\zeta
\end{array}\right)\right\rangle}=1-\left\langle R_{\lambda}(x, y) \zeta, \xi\right\rangle+O\left(\lambda^{-\frac{n-1}{2}}\right)
$$

and that the integral of $\left\langle R_{\lambda}(x, y) \eta, \xi\right\rangle$ against the Gaussian density above is 0 . This proves (97) for $i=3$, and completes the proof of Theorem 1 modulo the proof of (86)), which we now supply.

Proof of (86). By [8, Thm. 2], as $\lambda \rightarrow \infty$ we have for each $\gamma, \delta=1, \ldots, n$,

$$
\nabla_{x}^{\alpha}\left(T_{x, \lambda}\right)_{\gamma, \delta}=C_{\alpha} \lambda^{\alpha+2}\left(1+o_{\alpha}(1)\right) .
$$

Thus, the entries of $\nabla_{x}^{\alpha} T_{x, \lambda}^{-1 / 2}$ are bounded by a constant times $\lambda^{\alpha-1}$. Since

$$
\operatorname{Cov}\left(\psi_{\lambda}(x), \psi_{\lambda}(y)\right)=T_{x, \lambda}^{-1 / 2} \operatorname{Cov}\left(d \phi_{\lambda}(x), d \phi_{\lambda}(y)\right) T_{y, \lambda}^{-1 / 2},
$$

(86) is equivalent to showing that as $\lambda \rightarrow \infty$

$$
\left\|\lambda^{-|\alpha|-|\beta|} \nabla_{x}^{\alpha} \nabla_{y}^{\beta} \operatorname{Cov}\left(\lambda^{-1} d \phi_{\lambda}(x), \lambda^{-1} d \phi_{\lambda}(y)\right)_{\gamma, \delta}\right\|_{L^{2}(M \times M)}^{2}=O_{\alpha, \beta}\left(\lambda^{-n+1}\right),
$$

We will show more generally that if $P=\operatorname{Op}(p), Q=\mathrm{Op}(q)$ are pseudodifferential operators with orders $\operatorname{ord}(P), \operatorname{ord}(Q)$ acting on $M \times M$, then

$$
\left\langle P \Pi_{\lambda}, Q \Pi_{\lambda}\right\rangle_{L^{2}(M \times M)}=O\left(\lambda^{-n+1+\operatorname{ord}(P)+\operatorname{ord}(Q)}\right),
$$

as $\lambda \rightarrow \infty$, where the implied constant is uniform when $\|p\|_{L^{2}},\|q\|_{L^{2}}$ are bounded. We prove this by induction in $\operatorname{ord} P+\operatorname{ord} Q$. The base case is immediate since

$$
\left\langle\Pi_{\lambda}, \Pi_{\lambda}\right\rangle_{L^{2}(M \times M)}=\int_{M} \frac{\Pi_{\lambda}(x, x)}{\operatorname{dim} H_{\lambda}} d v_{g}(x)=\left(\operatorname{dim} H_{\lambda}\right)^{-1}=O\left(\lambda^{-n+1}\right) .
$$

Assume (100) is true for all operators whose orders sum to at most $\ell-1$ and consider $P, Q$ with $\operatorname{ord} P+\operatorname{ord} Q=\ell$. Then,

$$
\left\langle P \Pi_{\lambda}, Q \Pi_{\lambda}\right\rangle=\left\langle P \Delta_{x}^{1 / 2} \Pi_{\lambda}, \Delta_{x}^{-1 / 2} Q \Pi_{\lambda}\right\rangle+\left\langle\left[\Delta_{x}^{1 / 2}, P\right] \Pi_{\lambda}, \Delta_{x}^{-1 / 2} Q \Pi_{\lambda}\right\rangle .
$$

Note that $\operatorname{ord}\left(\left[\Delta_{x}^{1 / 2}, P\right]\right)+\operatorname{ord}\left(\Delta_{x}^{-1 / 2} Q\right) \leq \gamma-1$. Hence, the second term is $O\left(\lambda^{-n+\gamma}\right)$. Finally, $\Delta_{x}^{1 / 2} \Pi_{\lambda}=\lambda \Pi_{\lambda}+R_{\lambda} \Pi_{\lambda}$ where $R_{\lambda}=\mathrm{Op}\left(r_{\lambda}\right)$ is order 0 pseudodifferential 
operator with $\left\|r_{\lambda}\right\|_{L^{2}}$ uniformly bounded in $\lambda$. Therefore, $\left\langle P \Delta_{x}^{1 / 2} \Pi_{\lambda}, \Delta_{x}^{-1 / 2} Q \Pi_{\lambda}\right\rangle=$ $O\left(\lambda^{-n+1+\ell}\right)$, concluding the proof of (100).

\section{REFERENCES}

[1] J. Azaïs and M. Wschebor. Level sets and extrema of random processes and fields. John Wiley \&G Sons (2009).

[2] Y. Bonthonneau. A lower bound for the $\Theta$ function on manifolds without conjugate points. arXiv:1603.05697.

[3] P. Bérard. On the wave equation on a compact Riemannian manifold without conjugate points. Math. Z. 155 (1977), 249-276.

[4] P. Bérard. Volume des ensembles nodaux des fonctions propres du laplacien. Seinaire de theorie spectrale et geometrie. 1984. Vol 3, pp 1-9.

[5] M. Berry. Regular and irregular semi-classical wavefunctions. Journal of Physics A: Mathematical and General 1977. Vol 10, no 12.

[6] A. Bezubik, A. Dabrowska, A. Strasburger. A new derivation of the plane wave expansion into spherical harmonics and related Fourier transforms. Journal of Nonlinear Math. Phys. Vo1 11, Supplement (2004), 167-173.

[7] Y. Canzani and B. Hanin. Scaling Limit for the Kernel of the Spectral Projector and Remainder Estimates in the Pointwise Weyl Law. Analysis and Partial Differential Equations. Volume 8, no 7, (2015): pp 1707-1731.

[8] Y. Canzani, B. Hanin. $C^{\infty}$ scaling asymptotics for the spectral projector of the Laplacian. Journal of Geometric Analysis (in press). Preprint available: arXiv: 1602.00730 (2016).

[9] V. Cammarota and I. Wigman. Fluctuations of the total number of critical points of random spherical harmonics. Preprint available: arXiv:1510.00339 (2015).

[10] V. Cammarota, D. Marinucci and I. Wigman. On the distribution of the critical values of random spherical harmonics. The Journal of Geometric Analysis. (2014): pp 1-73.

[11] Y. Canzani and P. Sarnak. Topology and nesting of the zero set components of monochromatic random waves. Communications on Pure and Applied Mathematics. (2019). Volume 72, no. 2, 343-374.

[12] H. Donelly and C. Fefferman. Nodal sets of eigenfunctions on Reimannian manifolds. Inventiones mathematicae. Volume 93, no 1, (1988): pp 161-183.

[13] D. Gayet and J. Welschinger. Expected topology of random real algebraic submanifolds. Journal of the Institute of Mathematics of Jussieu. Volume 14, no 04, (2015): 673-702.

[14] D. Gayet and J. Welschinger. Betti numbers of random nodal sets of elliptic pseudo-differential operators. Preprint available: arXiv:1406.0934 (2014)

[15] D. Gayet and J. Welschinger. Universal components of random nodal sets. Communications in Mathematical Physics (2015): 1-21.

[16] L. Hörmander. The spectral function of an elliptic operator. Acta mathematica, Volume 121, no 1, (1968): 193-218.

[17] D. Jakobson and I. Polterovich. Estimates from Below for the Spectral Function and for the Remainder in Local Weyl? Law. Geometric Functional Analysis. (2007) 17: 806.

[18] B. Keeler. A logarithmic improvement in the two point Weyl Law for manifolds without conjugate points. Preprint available upon request.

[19] M. Krishnapur, P. Kurlberg, I. Wigman. Nodal length fluctuations for arithmetic random waves. Preprint available: arXiv:1111.2800 (2011).

[20] H. Kunita. Stochastic flows and stochastic differential equations. Cambridge university press, vol. 24, 1997.

[21] D. Marinucci, G. Peccati, M. Rossi and I. Wigman. Non-Universality of Nodal Length Distribution for Arithmetic Random Waves. Preprint available: arXiv:1508.00353 (2015).

[22] I. Nourdin, G. Peccati, and M. Rossi Nodal statistics of planar random waves. arXiv preprint arXiv:1708.02281 
[23] M. Sodin and F. Nazarov. Asymptotic laws for the spatial distribution and the number of connected components of zero sets of Gaussian random functions Preprint available: arXiv:1507.02017 (2016).

[24] J. Neuheisel. The asymptotic distribution of nodal sets on spheres. Diss. Johns Hopkins University (2010).

[25] L. Nicolaescu. Critical sets of random smooth functions on products of spheres . Preprint available: arXiv:1008.5085 (2010).

[26] Z. Rudnick and I. Wigman. On the volume of nodal sets for eigenfunctions of the Laplacian on the torus. Annales Henri Poincare Volume 9, no 1, (2008): pp. 109-130.

[27] Yu. Safarov. Asymptotics of the spectral function of a positive elliptic operator without a nontrapping condition. Funktsional. Anal. i Prilozhen. 22 (1988): no. 3, 53-65, 96 (Russian). English translation in Funct. Anal. Appl. Volume 22, no 3, (1988): pp. 213-223.

[28] P. Sarnak and I. Wigman. Topologies of Nodal Sets of Random Band-Limited Functions Communications on Pure and Applied Mathematics.72.2 (2019): 275-342.

[29] C. Sogge and S. Zelditch. Riemannian manifolds with maximal eigenfunction growth. Duke Mathematical Journal . Volume 114, no 3, (2002), pp 387 - 437.

[30] I. Wigman. On the distribution of the nodal sets of random spherical harmonics. Journal of Mathematical Physics. Volume 50, no 1,(2009)

[31] I. Wigman. Fluctuations of the nodal length of random spherical harmonics. Communications in Mathematical Physics. Volume 298, no 3, (2010) pp. 787-831.

[32] S. Zelditch. Real and complex zeros of Riemannian random waves. Contemporary Mathematics. Volume 14 (2009).

(Y. Canzani) Department of Mathematics, University of North Carolina, Chapel Hill, UNited STATES.

E-mail address: canzani@email.unc.edu

(B. Hanin) Department of Mathematics, Texas A\&M and Facebook Ai Research, NYC

E-mail address: bhanin@math.tamu.edu 